Article

\title{
An Algorithm for Fuzzy Negations Based-Intuitionistic Fuzzy Copula Aggregation Operators in Multiple Attribute Decision Making
}

\author{
Stylianos Giakoumakis and Basil Papadopoulos * (10 \\ Department of Civil Engineering Section of Mathematics and Informatics, Democritus University of Thrace, \\ 67100 Kimeria, Greece; sgiakoum@civil.duth.gr \\ * Correspondence: papadob@civil.duth.gr; Tel.: +30-2541079747
}

Received: 29 May 2020; Accepted: 23 June 2020; Published: 26 June 2020

check for updates

\begin{abstract}
In this paper, we develop a novel computation model of Intuitionistic Fuzzy Values with the usage of fuzzy negations and Archimedean copulas. This novel computation model's structure is based on the extension of the existing operations of intuitionistic fuzzy values with some classes of fuzzy negations. Many properties of the proposed operations are investigated and proved. Additionally, in this paper we introduce the concepts of intuitionistic fuzzy Archimedean copula weighted arithmetic and geometric aggregation operators based on fuzzy negations, including a further analysis of their properties. Finally, using a case study from an already published paper we found that our method has many advantages.
\end{abstract}

Keywords: intuitionistic fuzzy sets; fuzzy negations; copula; intuitionistic fuzzy Archimedean copula aggregation operators; multiple attribute decision making

\section{Introduction}

Atanassov [1] introduced the notion Intuitionistic Fuzzy Set (IFS), which generalizes the notion of Fuzzy Set proposed by Zadeh [2]. Based on the fact that IFS is appropriate for cases dealing with uncertainty and vagueness, many authors have applied them to decision making. In the case of multiple attribute decision making the operational laws of Intuitionistic Fuzzy Values (IFVs) propose an elementary and remarkable topic. Atanassov [1,3] introduced the basic operational laws and presented some of their properties. Beliakov et al. [4] developed some operations by using additive generators of the product t-norm. Zhao and Wei [5] proposed the intuitionistic fuzzy Einstein hybrid aggregation operators. Atanassov, Pasi and Yager [6] contributed to the case of multi-criteria group decision making, with the attributes being intuitionistic fuzzy numbers and the corresponding weights being crisp numerical values; their method is applied in the context of public relations and mass communication. Wang and Li [7] proposed the score function and the weighted score function methods. Ouyang and Pedrycz [8] proposed a model for intuitionistic fuzzy multi- attributes decision making that deals with the degrees of membership and nonmembership individually that is applied in multiple criteria supplier selection problems. Tao et al. [9] gave an intuitionistic fuzzy copula arithmetic aggregation operator in multi-attribute decision making. Liu and Li [10], extended power Bonferroni mean to interval-valued intuitionistic fuzzy numbers and applied it to multiple attribute group decision making in the evaluation of air quality. Seikh and Mandal [11], introduced intuitionistic fuzzy Dombi weighted averaging operator, intuitionistic fuzzy Dombi hybrid averaging operator, intuitionistic fuzzy Dombi weighted geometric operator, intuitionistic fuzzy Dombi ordered weighted geometric operator and intuitionistic fuzzy Dombi hybrid geometric operator. Xian, Guo and Chai [12], introduced an intuitionistic fuzzy linguistic-induced generalized ordered weighted averaging operator 
and made an application about taking targeted measures in poverty alleviation (TPA). Shi, Yang and Xiao [13], introduced the intuitionistic fuzzy power geometric Heronian mean operator and the weighted intuitionistic fuzzy power geometric Heronian mean operator. Zou, Chen and Fan [14], introduced the improved intuitionistic fuzzy weighted geometric operator of intuitionistic fuzzy values. For further contributions, references are provided [15-22].

Näther [23] states that copulas and t-norms often coincide. Nelsen [24] and Alsina et al. [25] contributed to the introduction and application of copulas. Beliakov et al. [26], has set copulas as conjunctive functions. In addition, the selection of the most suitable conjunctive function and how to fit additive generators for Archimedean t-norms and Archimedean copulas is depicted in Näther [23].

The present paper aims to investigate the intuitionistic fuzzy copula aggregation operators, with the combination of fuzzy negations, in order to develop multiple attribute decision making methods for IFVs. To accomplish that, the construction of novel operations for IFVs is essential. As a result, the combination of copulas and fuzzy negations [27] produces new operations, which allow us to construct novel intuitionistic fuzzy Archimedean copula weighted arithmetic and geometric aggregation operations with the usage of arithmetic and geometric means.

As a result, the remainder of the paper is organized as follows. Section 2 includes some basic concepts of copula theory, fuzzy negations and intuitionistic fuzzy sets and also our development on novel operations for IFVs with the combination of the above. The properties of the proposed operations are investigated. In Section 3 we provide the new intuitionistic fuzzy Archimedean copula weighted arithmetic and geometric aggregation operators, including a further analysis of their properties. In Section 4 we provide an algorithm to accomplish the multiple attribute decision making procedure. In Section 5 we provide a practical example to materialize the application of the proposed approach. In the discussion section, the results of our work are explained thoroughly and are compared with previous studies. In Section 7, we provide some concluding remarks and future research directions.

\section{Preliminaries}

This Section provides novel operations for IFVs, which are established through the concepts of copula, co-copula, Archimedean copula and fuzzy negations.

Sklar [28] presented the concept of copula, as a mathematical tool to combine probability distributions, framing the dependence structure within random variables with the usage of their CDFs.

Definition 1. [28] A 2-dimensional copula is a function with domain $[0,1] \times[0,1]$ and range $[0,1]$, grounded and 2-increasing, i.e., satisfies the following boundary and monotonicity conditions:

1. $C(x, 0)=C(0, y)=0$.

2. $C(x, 1)=x, C(1, y)=y$.

3. $C\left(x_{1}, y_{1}\right)+C\left(x_{2}, y_{2}\right)-C\left(x_{1}, y_{2}\right)-C\left(x_{2}, y_{1}\right) \geq 0$.

where, $x, y, x_{1}, x_{2}, y_{1}, y_{2} \in[0,1]$ and $x_{1} \leq x_{2}, y_{1} \leq y_{2}$.

Below, the definition of co-copula is given, according to Cherubini et al.'s [29] work:

Definition 2. [29] The co-copula of a copula is defined as:

$$
C^{*}(x, y)=1-C(1-x, 1-y)
$$

According to Nelsen [24] the co-copula is not a copula, but may be constructed by the usage of a copula. Fréchet [30] and Hoeffding [31] introduced the Fréchet-Hoeffding [24] bounds of copulas for any $x, y \in[0,1], \max \{x+y-1,0\} \leq C(x, y) \leq \min \{x, y\}$. At this point, the method follows the definition of Archimedean copulas, which may be constructed with significant ease and ensure some remarkable properties. 
Definition 3. [24] Let $\varphi$ be a continuous, strictly decreasing function on $[0,1] \rightarrow[0,+\infty]$ with $\varphi(1)=0$ and $\varphi^{[-1]}$ the pseudo-inverse of $\varphi$, which is defined as:

$$
\varphi^{[-1]}(x)= \begin{cases}\varphi^{-1}(x), & 0 \leq x \leq \varphi(0), \\ 0, & \varphi(0) \leq x \leq+\infty,\end{cases}
$$

Then, $C:[0,1]^{2} \rightarrow[0,1], C(x, y)=\varphi^{[-1]}(\varphi(x)+\varphi(y))$ corresponds to the conditions of Definition 1 , hence $C$ is a copula and $C$ is called Archimedean copula.

In the case, where $C$ is strictly increasing, $\varphi(0)=+\infty$ and $\varphi^{[-1]}=\varphi^{-1}$, then $C(x, y)=\varphi^{-1}(\varphi(x)+$ $\varphi(y))$. In that case $\varphi$ is called strict generator and $C$ is called strict Archimedean copula.

In Table 1, some Archimedean copulas are listed.

Table 1. In ref. [24] Archimedean Copulas.

\begin{tabular}{cccc}
\hline Name & $\boldsymbol{\varphi}(\boldsymbol{t})$ & $\boldsymbol{C}(\boldsymbol{x}, \boldsymbol{y})$ & Parameter \\
\hline Gumbel & $(-\ln t)^{\theta}$ & $\exp \left(-\left[(-\ln x)^{\theta}+(-\ln y)^{\theta}\right]^{\frac{1}{\theta}}\right)$ & $\theta \geq 1$ \\
Clayton & $t^{-\theta}-1$ & $\left(x^{-\theta}+y^{-\theta}-1\right)^{-\frac{1}{\theta}}$ & $\theta>0$ \\
Ali-Mikhail-Haq & $\ln \frac{1-\theta(1-t)}{t}$ & $\frac{x y}{1-\theta(1-x)(1-y)}$ & $-1 \leq \theta<1$ \\
Joe & $-\ln \left[1-(1-t)^{\theta}\right]$ & $1-\left[(1-x)^{\theta}+(1-y)^{\theta}-(1-x)^{\theta}(1-y)^{\theta}\right]^{\frac{1}{\theta}}$ & $\theta \geq 1$ \\
\hline
\end{tabular}

The study of the applications of copulas in fuzzy sets is of great importance, given that specific copulas are t-norms and specific co-copulas are t-conorms and vice versa. Moreover, Näther [23] mentioned in his work that the combination of probabilistic information or fuzzy information hardly matters. Below, the Atanassov's [1] definition for Intuitionistic Fuzzy Sets is provided:

Definition 4. [1] Let X be a reference set, an Intuitionistic Fuzzy Set (IFS) A on X is defined as:

$$
A=\left\{\left\langle x, \mu_{A}(x), v_{A}(x)\right\rangle \mid x \in X\right\},
$$

where, the functions $\mu_{A}(x)$ and $v_{A}(x)$ denote the degrees of membership and non-membership of the element $x \in X$ to the set $A$, respectively with:

$0 \leq \mu_{A}(x) \leq 1,0 \leq v_{A}(x) \leq 1,0 \leq \mu_{A}(x)+v_{A}(x) \leq 1$ and $\pi_{A}(x)=1-\mu_{A}(x)-v_{A}(x)$ is called degree of indeterminacy of $x$ to A. Additionally, $\alpha=\left\langle\mu_{\alpha}, v_{\alpha}\right\rangle$ is called Intuitionistic Fuzzy Value (IFV) and the set of all IFVs is $V$.

Definition 5. [32] A decreasing function $n:[0,1] \rightarrow[0,1]$ is called a fuzzy negation if $n(0)=1$ and $n(1)=0$. A fuzzy negation $n$ is called: Strict, if it is strictly decreasing and continuous and Strong, if it is an involution, i.e., $n(n(x))=x, \forall x \in[0,1]$.

Table 2, proposes two parametric families of fuzzy negations:

Table 2. In ref. [32] Fuzzy Negations.

\begin{tabular}{ccc}
\hline Name & Fuzzy Negation & Parameter \\
\hline Sugeno class & $n_{\lambda}(x)=\frac{1-x}{1+\lambda x}$ & $\lambda \in(-1, \infty)$ \\
Yager class & $n_{\lambda}(x)=\left(1-x^{\lambda}\right)^{1 / \lambda}$ & $\lambda \in(0, \infty)$ \\
\hline
\end{tabular}

Moreover, the operations of IFVs in Tao et al.'s [9] work, are being extended through the adaptation of fuzzy negations. 
Definition 6. Let $\alpha=\left\langle\mu_{\alpha}, v_{\alpha}\right\rangle$ and $\beta=\left\langle\mu_{\beta}, v_{\beta}\right\rangle$ be two IFVs and $k \geq 0$ a parameter.

1. Addition operation

$$
\alpha \oplus_{C} \beta=\left\langle C_{\lambda}^{*}\left(\mu_{\alpha}, \mu_{\beta}\right), C\left(v_{\alpha}, v_{\beta}\right)\right\rangle=\left\langle n_{\lambda}\left(\varphi^{-1}\left[\varphi\left(n_{\lambda}\left(\mu_{\alpha}\right)\right)+\varphi\left(n_{\lambda}\left(\mu_{\beta}\right)\right)\right]\right), \varphi^{-1}\left[\varphi\left(v_{\alpha}\right)+\varphi\left(v_{\beta}\right)\right]\right\rangle .
$$

2. Multiplication operation

$$
\alpha \otimes_{C} \beta=\left\langle C\left(\mu_{\alpha}, \mu_{\beta}\right), C_{\lambda}^{*}\left(v_{\alpha}, v_{\beta}\right)\right\rangle=\left\langle\varphi^{-1}\left[\varphi\left(\mu_{\alpha}\right)+\varphi\left(\mu_{\beta}\right)\right], n_{\lambda}\left(\varphi^{-1}\left[\varphi\left(n_{\lambda}\left(v_{\alpha}\right)\right)+\varphi\left(n_{\lambda}\left(v_{\beta}\right)\right)\right]\right)\right\rangle .
$$

3. Scalar Multiplication operation

$$
k \alpha=\left\langle n_{\lambda}\left(\varphi^{-1}\left[k \varphi\left(n_{\lambda}\left(\mu_{\alpha}\right)\right)\right]\right), \varphi^{-1}\left[k \varphi\left(v_{\alpha}\right)\right]\right\rangle .
$$

4. Power operation

$$
\alpha^{k}=\left\langle\varphi^{-1}\left[k \varphi\left(\mu_{\alpha}\right)\right], n_{\lambda}\left(\varphi^{-1}\left[k \varphi\left(n_{\lambda}\left(v_{\alpha}\right)\right)\right]\right)\right\rangle .
$$

By replacing the $\lambda$ parameter with the appropriate value, the Tao et al.'s [9] operations of IFVs are produced. Moreover, a further investigation is held for the properties of the proposed operations.

Theorem 1. Let $\alpha=\left\langle\mu_{\alpha}, v_{\alpha}\right\rangle$ and $\beta=\left\langle\mu_{\beta}, v_{\beta}\right\rangle$ be two IFVs and $k \geq 0$ a real valued parameter. The novel operations of IFVs are closed or their values are also IFVs, i.e., $\alpha \oplus_{C} \beta, \alpha \otimes_{C} \beta, k \alpha, \alpha^{k} \in V$.

Proof of Theorem 1. We have $\mu_{\alpha} \in[0,1]$ and $0 \leq n_{\lambda}\left(\mu_{\alpha}\right) \leq 1$. Since $\varphi$ is strictly decreasing then:

$$
\varphi(1) \leq \varphi\left(n_{\lambda}\left(\mu_{\alpha}\right)\right) \leq \varphi(0) \Leftrightarrow 0 \leq \varphi\left(n_{\lambda}\left(\mu_{\alpha}\right)\right) \leq+\infty,
$$

Similarly, as $\mu_{\beta} \in[0,1]$ we get:

$$
0 \leq \varphi\left(n_{\lambda}\left(\mu_{\beta}\right)\right) \leq+\infty,
$$

We add Equations (4) and (5) and we get:

$$
0 \leq \varphi\left(n_{\lambda}\left(\mu_{\alpha}\right)\right)+\varphi\left(n_{\lambda}\left(\mu_{\beta}\right)\right) \leq+\infty,
$$

Based on the fact that $\varphi^{-1}$ is also strictly decreasing, we obtain:

$$
\begin{gathered}
\varphi^{-1}(+\infty) \leq \varphi^{-1}\left(\varphi\left(n_{\lambda}\left(\mu_{\alpha}\right)\right)+\varphi\left(n_{\lambda}\left(\mu_{\beta}\right)\right)\right) \leq \varphi^{-1}(0) \Leftrightarrow \\
0 \leq \varphi^{-1}\left(\varphi\left(n_{\lambda}\left(\mu_{\alpha}\right)\right)+\varphi\left(n_{\lambda}\left(\mu_{\beta}\right)\right)\right) \leq 1 \Leftrightarrow \\
0 \leq n_{\lambda}\left(\varphi^{-1}\left(\varphi\left(n_{\lambda}\left(\mu_{\alpha}\right)\right)+\varphi\left(n_{\lambda}\left(\mu_{\beta}\right)\right)\right) \leq 1 \Leftrightarrow\right. \\
0 \leq C_{\lambda}^{*}\left(\mu_{\alpha}, \mu_{\beta}\right) \leq 1 .
\end{gathered}
$$

Morover, by Definition 1 , as $v_{\alpha}, v_{\beta} \in[0,1]$,

$$
0 \leq C\left(v_{\alpha}, v_{\beta}\right) \leq 1
$$

holds.

In addition:

$$
0 \leq C_{\lambda}^{*}\left(\mu_{\alpha}, \mu_{\beta}\right)+C\left(v_{\alpha}, v_{\beta}\right) \leq 1 .
$$

Thus, the result of the operation is:

$$
\alpha \oplus_{C} \beta \in V
$$


As Equation (3) is concerned:

$$
k \alpha=\left\langle n_{\lambda}\left(\varphi^{-1}\left[k \varphi\left(n_{\lambda}\left(\mu_{\alpha}\right)\right)\right]\right), \varphi^{-1}\left[k \varphi\left(v_{\alpha}\right)\right]\right\rangle
$$

For $k=1$ we get:

$$
\begin{gathered}
\alpha=\left\langle n_{\lambda}\left(\varphi^{-1}\left[\varphi\left(n_{\lambda}\left(\mu_{\alpha}\right)\right)\right]\right), \varphi^{-1}\left[\varphi\left(v_{\alpha}\right)\right]\right\rangle \Leftrightarrow \\
\alpha=\left\langle n_{\lambda}\left(n_{\lambda}\left(\mu_{\alpha}\right)\right), v_{\alpha}\right\rangle \Leftrightarrow \\
\alpha=\left\langle\mu_{\alpha}, v_{\alpha}\right\rangle,
\end{gathered}
$$

that holds.

For $k=2$ we get:

$$
\begin{gathered}
\alpha \oplus_{C} \alpha=\left\langle n_{\lambda}\left(\varphi^{-1}\left[\varphi\left(n_{\lambda}\left(\mu_{\alpha}\right)\right)+\varphi\left(n_{\lambda}\left(\mu_{\alpha}\right)\right)\right]\right), \varphi^{-1}\left[\varphi\left(v_{\alpha}\right)+\varphi\left(v_{\alpha}\right)\right]\right\rangle \Leftrightarrow \\
\alpha \oplus_{C} \alpha=\left\langle n_{\lambda}\left(\varphi^{-1}\left[2 \varphi\left(n_{\lambda}\left(\mu_{\alpha}\right)\right)\right]\right), \varphi^{-1}\left[2 \varphi\left(v_{\alpha}\right)\right]\right\rangle=2 \alpha,
\end{gathered}
$$

that holds.

We assume that for $k=n$ holds. Then for $k=n+1$ :

$$
\begin{gathered}
k \alpha=(n+1) \alpha=n \alpha \oplus_{C} \alpha=\left\langle n_{\lambda}\left[\varphi^{-1}\left[n \varphi\left(n_{\lambda}\left(\mu_{\alpha}\right)\right)\right]\right], \varphi^{-1}\left[n \varphi\left(v_{\alpha}\right)\right]\right\rangle \oplus_{C}\left\langle\mu_{\alpha}, v_{\alpha}\right\rangle \Leftrightarrow \\
k \alpha=\left\langle n_{\lambda}\left(\varphi^{-1}\left[\varphi\left(n_{\lambda}\left(n_{\lambda}\left[\varphi^{-1}\left[n \varphi\left(n_{\lambda}\left(\mu_{\alpha}\right)\right)\right]\right]\right)\right)\right]\right)+\varphi\left(n_{\lambda}\left(\mu_{\alpha}\right)\right), \varphi^{-1}\left(\varphi\left(\varphi^{-1}\left(n \varphi\left(v_{\alpha}\right)\right)\right)+\varphi\left(v_{\alpha}\right)\right)\right\rangle \Leftrightarrow \\
k \alpha=\left\langle n_{\lambda}\left(\varphi^{-1}\left[n \varphi\left(n_{\lambda}\left(\mu_{\alpha}\right)\right)\right]\right)+\varphi\left(n_{\lambda}\left(\mu_{\alpha}\right)\right), \varphi^{-1}\left((n+1) \varphi\left(v_{\alpha}\right)\right)\right\rangle \Leftrightarrow \\
k \alpha=\left\langle n_{\lambda}\left(\varphi^{-1}\left[(n+1) \varphi\left(n_{\lambda}\left(\mu_{\alpha}\right)\right)\right]\right), \varphi^{-1}\left((n+1) \varphi\left(v_{\alpha}\right)\right)\right\rangle=(n+1) \alpha .
\end{gathered}
$$

Hence, by mathematical induction the proof is complete. The proofs that the rest of the operations belong to the set $V$ are similar.

Next we investigate the properties of the proposed operations:

Theorem 2. Let $\alpha=\left\langle\mu_{\alpha}, v_{\alpha}\right\rangle \in V, \beta=\left\langle\mu_{\beta}, v_{\beta}\right\rangle \in V$ and real valued parameters $k, k_{1}, k_{2} \geq 0$, then:

1. $\alpha \oplus_{C} \beta=\beta \oplus_{C} \alpha$.

2. $\alpha \otimes_{C} \beta=\beta \otimes_{C} \alpha$.

3. $k\left(\alpha \oplus_{C} \beta\right)=k \alpha \oplus_{C} k \beta$.

4. $\left(\alpha \otimes_{\mathcal{C}} \beta\right)^{k}=\alpha^{k} \otimes_{\mathcal{C}} \beta^{k}$.

5. $k_{1} \alpha \oplus_{C} k_{2} \alpha=\left(k_{1}+k_{2}\right) \alpha$.

6. $\alpha^{k_{1}} \otimes_{C} \alpha^{k_{2}}=\alpha^{k_{1}+k_{2}}$.

7. $n_{\lambda}(\alpha) \oplus_{C} n_{\lambda}(\beta)=n_{\lambda}\left(\alpha \otimes_{C} \beta\right)$.

8. $n_{\lambda}(\alpha) \otimes_{C} n_{\lambda}(\beta)=n_{\lambda}\left(\alpha \oplus_{C} \beta\right)$.

9. $k n_{\lambda}(\alpha)=n_{\lambda}\left(\alpha^{k}\right)$.

10. $\left(n_{\lambda}(\alpha)\right)^{k}=n_{\lambda}(k \alpha)$.

Proof of Theorem 2. The cases 1 and 2 are obvious, because of Archimedean copulas and the corresponding co-copulas commutativity. 
1. $\alpha \oplus_{C} \beta=\left\langle C_{\lambda}^{*}\left(\mu_{\alpha}, \mu_{\beta}\right), C\left(v_{\alpha}, v_{\beta}\right)\right\rangle=\left\langle n_{\lambda}\left(\varphi^{-1}\left[\varphi\left(n_{\lambda}\left(\mu_{\alpha}\right)\right)+\varphi\left(n_{\lambda}\left(\mu_{\beta}\right)\right)\right]\right), \varphi^{-1}\left[\varphi\left(v_{\alpha}\right)+\varphi\left(v_{\beta}\right)\right]\right\rangle=$ $=\left\langle n_{\lambda}\left(\varphi^{-1}\left[\varphi\left(n_{\lambda}\left(\mu_{\beta}\right)\right)+\varphi\left(n_{\lambda}\left(\mu_{\alpha}\right)\right)\right]\right), \varphi^{-1}\left[\varphi\left(v_{\beta}\right)+\varphi\left(v_{\alpha}\right)\right]\right\rangle=\left\langle C_{\lambda}^{*}\left(\mu_{\beta}, \mu_{\alpha}\right), C\left(v_{\beta}, v_{\alpha}\right)\right\rangle=\beta \oplus_{C} \alpha$.

2. $\alpha \otimes_{C} \beta=\left\langle C\left(\mu_{\alpha}, \mu_{\beta}\right), C_{\lambda}^{*}\left(v_{\alpha}, v_{\beta}\right)\right\rangle=\left\langle C\left(\mu_{\beta}, \mu_{\alpha}\right), C_{\lambda}^{*}\left(v_{\beta}, v_{\alpha}\right)\right\rangle=\beta \otimes_{C} \alpha$.

3. $k\left(\alpha \oplus_{C} \beta\right)=\left\langle n_{\lambda}\left(\varphi^{-1}\left[k \varphi\left(n_{\lambda}\left(n_{\lambda}\left(\varphi^{-1}\left[\varphi\left(n_{\lambda}\left(\mu_{\alpha}\right)\right)+\varphi\left(n_{\lambda}\left(\mu_{\beta}\right)\right)\right]\right)\right)\right]\right), \varphi^{-1}\left[k \varphi\left(\varphi^{-1}\left[\varphi\left(v_{\alpha}\right)+\varphi\left(v_{\beta}\right)\right]\right)\right]\right\rangle \Leftrightarrow\right.$ $k\left(\alpha \oplus_{C} \beta\right)=\left\langle n_{\lambda}\left(\varphi^{-1}\left[k\left[\varphi\left(n_{\lambda}\left(\mu_{\alpha}\right)\right)+\varphi\left(n_{\lambda}\left(\mu_{\beta}\right)\right)\right]\right]\right), \varphi^{-1}\left[k\left[\varphi\left(v_{\alpha}\right)+\varphi\left(v_{\beta}\right)\right]\right]\right\rangle \Leftrightarrow$ $k\left(\alpha \oplus_{C} \beta\right)=\left\langle n_{\lambda}\left(\varphi^{-1}\left[k \varphi\left(n_{\lambda}\left(\mu_{\alpha}\right)\right)+k \varphi\left(n_{\lambda}\left(\mu_{\beta}\right)\right)\right]\right), \varphi^{-1}\left[k \varphi\left(v_{\alpha}\right)+k \varphi\left(v_{\beta}\right)\right]\right\rangle \Leftrightarrow$ $k\left(\alpha \oplus_{C} \beta\right)=\left\langle n_{\lambda}\left(\varphi^{-1}\left[\varphi\left(\varphi^{-1}\left(k \varphi\left(n_{\lambda}\left(\mu_{\alpha}\right)\right)\right)\right)+\varphi\left(\varphi^{-1}\left(k \varphi\left(n_{\lambda}\left(\mu_{\beta}\right)\right)\right)\right)\right]\right), \varphi^{-1}\left[\varphi\left(\varphi^{-1}\left(k \varphi\left(v_{\alpha}\right)\right)\right)+\varphi\left(\varphi^{-1}\left(k \varphi\left(v_{\beta}\right)\right)\right)\right]\right\rangle \Leftrightarrow$ $\left.\left.\left.k\left(\alpha \oplus_{C} \beta\right)=\left\langle n_{\lambda}\left(\varphi^{-1}\left[\phi n_{\lambda}\left(n_{\lambda}\left(\varphi^{-1}\left(k \phi\left(n_{\lambda}\left(\mu_{\alpha}\right)\right)\right)\right)\right)\right)+\varphi\left(n_{\lambda}\left(n_{\lambda}\left(\varphi^{-1}\left(k \phi n_{\lambda}\left(\mu_{\beta}\right)\right)\right)\right)\right)\right)\right], \varphi^{-1}\left[\phi\left(\varphi^{-1}\left(k \phi v_{\alpha}\right)\right)\right)+\phi\left(\varphi^{-1}\left(k \phi v_{\beta}\right)\right)\right)\right]\right) \Leftrightarrow$ $k\left(\alpha \oplus_{C} \beta\right)=k \alpha \oplus_{C} k \beta$.

4. $\quad\left(\alpha \otimes_{C} \beta\right)^{k}=\left\langle\varphi^{-1}\left[k \varphi\left(\varphi^{-1}\left[\varphi\left(\mu_{\alpha}\right)+\varphi\left(\mu_{\beta}\right)\right]\right)\right], n_{\lambda}\left(\varphi^{-1}\left[k \varphi\left(n_{\lambda}\left(n_{\lambda}\left(\varphi^{-1}\left[\varphi\left(n_{\lambda}\left(v_{\alpha}\right)\right)+\varphi\left(n_{\lambda}\left(v_{\beta}\right)\right)\right]\right)\right)\right)\right]\right)\right\rangle=$ $=\left\langle\varphi^{-1}\left[k\left[\varphi\left(\mu_{\alpha}\right)+\varphi\left(\mu_{\beta}\right)\right]\right], n_{\lambda}\left(\varphi^{-1}\left[k\left[\varphi\left(n_{\lambda}\left(v_{\alpha}\right)\right)+\varphi\left(n_{\lambda}\left(v_{\beta}\right)\right)\right]\right]\right)\right\rangle=\left\langle\varphi^{-1}\left[k \varphi\left(\mu_{\alpha}\right)+k \varphi\left(\mu_{\beta}\right)\right]\right], n_{\lambda}\left(\varphi^{-1}\left[k \varphi\left(n_{\lambda}\left(v_{\alpha}\right)\right)+\right.\right.$ $\left.\left.\left.+k \varphi\left(n_{\lambda}\left(v_{\beta}\right)\right)\right]\right)\right\rangle=\left\langle\varphi^{-1}\left[\varphi\left(\varphi^{-1}\left(k \varphi\left(\mu_{\alpha}\right)\right)\right)+\varphi\left(\varphi^{-1}\left(k \varphi\left(\mu_{\beta}\right)\right)\right)\right], n_{\lambda}\left(\varphi^{-1}\left[\varphi\left(n_{\lambda}\left(n_{\lambda}\left(\varphi^{-1}\left(k \varphi\left(n_{\lambda}\left(v_{\alpha}\right)\right)\right)\right)\right)\right)+\right.\right.\right.$ $\left.\left.\left.+\varphi\left(n_{\lambda}\left(n_{\lambda}\left(\varphi^{-1}\left(k \varphi\left(n_{\lambda}\left(v_{\beta}\right)\right)\right)\right)\right)\right)\right]\right)\right\rangle=\alpha^{k} \otimes_{C} \beta^{k}$.

5. $\quad k_{1} \alpha \oplus_{C} k_{2} \alpha=\left\langle n_{\lambda}\left(\varphi^{-1}\left[k_{1} \varphi\left(n_{\lambda}\left(\mu_{\alpha}\right)\right)\right]\right), \varphi^{-1}\left[k_{1} \varphi\left(v_{\alpha}\right)\right]\right\rangle \oplus_{C}\left\langle n_{\lambda}\left(\varphi^{-1}\left[k_{2} \varphi\left(n_{\lambda}\left(\mu_{\alpha}\right)\right)\right]\right), \varphi^{-1}\left[k_{2} \varphi\left(v_{\alpha}\right)\right]\right\rangle=$ $=\left\langle n_{\lambda}\left(\varphi^{-1}\left[\varphi\left(n_{\lambda}\left(n_{\lambda}\left(\varphi^{-1}\left[k_{1} \varphi\left(n_{\lambda}\left(\mu_{\alpha}\right)\right)\right]\right)\right)\right)+\varphi\left(n_{\lambda}\left(n_{\lambda}\left(\varphi^{-1}\left[k_{2} \varphi\left(n_{\lambda}\left(\mu_{\alpha}\right)\right)\right]\right)\right)\right)\right]\right), \varphi^{-1}\left[\varphi\left(\varphi^{-1}\left[k_{1} \varphi\left(v_{\alpha}\right)\right]\right)+\varphi\left(\varphi^{-1}\left[k_{2} \varphi\left(v_{\alpha}\right)\right]\right)\right]\right\rangle=$ $=\left\langle n_{\lambda}\left(\varphi^{-1}\left[k_{1} \varphi\left(n_{\lambda}\left(\mu_{\alpha}\right)\right)+k_{2} \varphi\left(n_{\lambda}\left(\mu_{\alpha}\right)\right)\right]\right), \varphi^{-1}\left[k_{1} \varphi\left(v_{\alpha}\right)+k_{2} \varphi\left(v_{\alpha}\right)\right]\right\rangle=\left\langle n_{\lambda}\left(\varphi^{-1}\left[\left(k_{1}+k_{2}\right) \varphi\left(n_{\lambda}\left(\mu_{\alpha}\right)\right)\right]\right)\right.$, , $\left.\varphi^{-1}\left[\left(k_{1}+k_{2}\right) \varphi\left(v_{\alpha}\right)\right]\right\rangle=\left(k_{1}+k_{2}\right) \alpha$.

6. $\quad \alpha^{k_{1}} \otimes_{C} \alpha^{k_{2}}=\left\langle\varphi^{-1}\left[k_{1} \varphi\left(\mu_{\alpha}\right)\right], n_{\lambda}\left(\varphi^{-1}\left[k_{1} \varphi\left(n_{\lambda}\left(v_{\alpha}\right)\right)\right]\right)\right\rangle \otimes_{C}\left\langle\varphi^{-1}\left[k_{2} \varphi\left(\mu_{\alpha}\right)\right], n_{\lambda}\left(\varphi^{-1}\left[k_{2} \varphi\left(n_{\lambda}\left(v_{\alpha}\right)\right)\right]\right)\right\rangle=$ $=\left\langle\varphi^{-1}\left[\varphi\left(\varphi^{-1}\left[k_{1} \varphi\left(\mu_{\alpha}\right)\right]\right)+\varphi\left(\varphi^{-1}\left[k_{2} \varphi\left(\mu_{\alpha}\right)\right]\right)\right], n_{\lambda}\left(\varphi^{-1}\left[\varphi\left(n_{\lambda}\left(n_{\lambda}\left(\varphi^{-1}\left[k_{1} \varphi\left(n_{\lambda}\left(v_{\alpha}\right)\right)\right]\right)+\varphi\left(n_{\lambda}\left(n_{\lambda}\left(\varphi^{-1}\left[k_{2} \varphi\left(n_{\lambda}\left(v_{\alpha}\right)\right)\right]\right)\right)\right]\right)\right\rangle=\right.\right.\right.$ $=\left\langle\varphi^{-1}\left[k_{1} \varphi\left(\mu_{\alpha}\right)+k_{2} \varphi\left(\mu_{\alpha}\right)\right], n_{\lambda}\left(\varphi^{-1}\left[k_{1} \varphi\left(n_{\lambda}\left(v_{\alpha}\right)\right)+k_{2} \varphi\left(n_{\lambda}\left(v_{\alpha}\right)\right)\right]\right)\right\rangle=\left\langle\varphi^{-1}\left[\left(k_{1}+k_{2}\right) \varphi\left(\mu_{\alpha}\right)\right]\right.$, ,$\left.n_{\lambda}\left(\varphi^{-1}\left[\left(k_{1}+k_{2}\right) \varphi\left(n_{\lambda}\left(v_{\alpha}\right)\right)\right]\right)\right\rangle=\alpha^{k_{1}+k_{2}}$.

7. $n_{\lambda}(\alpha) \oplus_{C} n_{\lambda}(\beta)=\left\langle n_{\lambda}\left(\varphi^{-1}\left[\varphi\left(n_{\lambda}\left(n_{\lambda}\left(\mu_{\alpha}\right)\right)\right)+\varphi\left(n_{\lambda}\left(n_{\lambda}\left(\mu_{\beta}\right)\right)\right)\right]\right), \varphi^{-1}\left[\varphi\left(n_{\lambda}\left(v_{\alpha}\right)\right)+\varphi\left(n_{\lambda}\left(v_{\beta}\right)\right)\right]\right\rangle=$ $=\left\langle n_{\lambda}\left(\varphi^{-1}\left[\varphi\left(\mu_{\alpha}\right)+\varphi\left(\mu_{\beta}\right)\right]\right), \varphi^{-1}\left[\varphi\left(n_{\lambda}\left(v_{\alpha}\right)\right)+\varphi\left(n_{\lambda}\left(v_{\beta}\right)\right)\right]\right\rangle=n_{\lambda}\left(\left\langle\varphi^{-1}\left[\varphi\left(\mu_{\alpha}\right)+\varphi\left(\mu_{\beta}\right)\right], n_{\lambda}\left(\varphi^{-1}\left[\varphi\left(n_{\lambda}\left(v_{\alpha}\right)\right)+\right.\right.\right.\right.$ $\left.\left.\left.\left.+\varphi\left(n_{\lambda}\left(v_{\beta}\right)\right)\right]\right)\right\rangle\right)=n_{\lambda}\left(\alpha \otimes_{C} \beta\right)$.

8. $\quad n_{\lambda}(\alpha) \otimes_{C} n_{\lambda}(\beta)=\left\langle\varphi^{-1}\left[\varphi\left(n_{\lambda}\left(\mu_{\alpha}\right)\right)+\varphi\left(n_{\lambda}\left(\mu_{\beta}\right)\right)\right], n_{\lambda}\left(\varphi^{-1}\left[\varphi\left(n_{\lambda}\left(n_{\lambda}\left(v_{\alpha}\right)\right)\right)+\varphi\left(n_{\lambda}\left(n_{\lambda}\left(v_{\beta}\right)\right)\right)\right]\right)\right\rangle=$ $=\left\langle\varphi^{-1}\left[\varphi\left(n_{\lambda}\left(\mu_{\alpha}\right)\right)+\varphi\left(n_{\lambda}\left(\mu_{\beta}\right)\right)\right], n_{\lambda}\left(\varphi^{-1}\left[\varphi\left(v_{\alpha}\right)+\varphi\left(v_{\beta}\right)\right]\right)\right\rangle=n_{\lambda}\left(n_{\lambda}\left(\varphi^{-1}\left[\varphi\left(n_{\lambda}\left(\mu_{\alpha}\right)\right)+\varphi\left(n_{\lambda}\left(\mu_{\beta}\right)\right)\right]\right), \varphi^{-1}\left[\varphi v_{\alpha}\right)+\right.$ $\left.\left.\left.+\varphi\left(v_{\beta}\right)\right]\right)\right)=n_{\lambda}\left(\alpha \oplus_{C} \beta\right)$.

9. $\quad k n_{\lambda}(\alpha)=k\left\langle n_{\lambda}\left(\mu_{\alpha}\right), n_{\lambda}\left(v_{\alpha}\right)\right\rangle=\left\langle n_{\lambda}\left(\varphi^{-1}\left[k \varphi\left(n_{\lambda}\left(n_{\lambda}\left(\mu_{\alpha}\right)\right)\right)\right]\right), \varphi^{-1}\left[k \varphi\left(n_{\lambda}\left(v_{\alpha}\right)\right)\right]\right\rangle=\left\langle n_{\lambda}\left(\varphi^{-1}\left[k \varphi\left(\mu_{\alpha}\right)\right]\right), \varphi^{-1}\left[k \varphi\left(n_{\lambda}\left(v_{\alpha}\right)\right)\right]\right\rangle=$ $=n_{\lambda}\left(\left\langle\varphi^{-1}\left[k \phi\left(\mu_{\alpha}\right)\right], n_{\lambda}\left(\varphi^{-1}\left[k \phi\left(n_{\lambda}\left(v_{\alpha}\right)\right)\right]\right)\right)\right)=n_{\lambda}\left(\alpha^{k}\right)$.

10. $\quad\left(n_{\lambda}(\alpha)\right)^{k}=\left\langle\varphi^{-1}\left[k \varphi\left(n_{\lambda}\left(\mu_{\alpha}\right)\right)\right], n_{\lambda}\left(\varphi^{-1}\left[k \varphi\left(n_{\lambda}\left(n_{\lambda}\left(v_{\alpha}\right)\right)\right)\right]\right)\right\rangle=\left\langle\varphi^{-1}\left[k \varphi\left(n_{\lambda}\left(\mu_{\alpha}\right)\right)\right], n_{\lambda}\left(\varphi^{-1}\left[k \varphi\left(v_{\alpha}\right)\right]\right)\right\rangle=$ $=n_{\lambda}\left(\left\langle n_{\lambda}\left(\varphi^{-1}\left[k \varphi\left(n_{\lambda}\left(\mu_{\alpha}\right)\right)\right]\right), \varphi^{-1}\left[k \varphi\left(v_{\alpha}\right)\right]\right)\right)=n_{\lambda}(k \alpha)$.

In order to achieve the derivation of different operations for IFVs, the usage of generator functions from Table 1 and of fuzzy negations from Table 2, is accomplished. Those operations are listed in Table 3: 
Table 3. Operations for intuitionistic fuzzy values (IFVs) via Sugeno Fuzzy Negation.

\begin{tabular}{|c|c|c|}
\hline Name & Operations & Formulas \\
\hline Gumbel & Addition operation & $\left\langle\frac{1-\exp \left(-\left[\left(-\ln \left(\frac{1-\mu_{\alpha}}{1+\lambda \mu_{\alpha}}\right)\right)^{\theta}+\left(-\ln \left(\frac{1-\mu_{\beta}}{1+\lambda \mu_{\beta}}\right)\right)^{\theta}\right]^{\frac{1}{\theta}}\right)}{1+\lambda \exp \left(-\left[\left(-\ln \left(\frac{1-\mu_{\alpha}}{1+\lambda \mu_{\alpha}}\right)\right)^{\theta}+\left(-\ln \left(\frac{1-\mu_{\beta}}{1+\lambda \mu_{\beta}}\right)\right)^{\theta}\right]^{\frac{1}{\theta}}\right)}, \exp \left(-\left[\left(-\ln v_{\alpha}\right)^{\theta}+\left(-\ln v_{\beta}\right)^{\theta}\right]^{\frac{1}{\theta}}\right)\right\rangle$ \\
\hline & Multiplication operation & $\left\langle\exp \left(-\left[\left(-\ln \mu_{\alpha}\right)^{\theta}+\left(-\ln \mu_{\beta}\right)^{\theta}\right]^{\frac{1}{\theta}}\right), \frac{1-\exp \left(-\left[\left(-\ln \left(\frac{1-v_{\alpha}}{1+\lambda v_{\alpha}}\right)\right)^{\theta}+\left(-\ln \left(\frac{1-v_{\beta}}{1+\lambda v_{\beta}}\right)\right)^{\theta}\right]^{\frac{1}{\theta}}\right)}{1+\lambda \exp \left(-\left[\left(-\ln \left(\frac{1-v_{\alpha}}{1+\lambda v_{\alpha}}\right)\right)^{\theta}+\left(-\ln \left(\frac{1-v_{\beta}}{1+\lambda v_{\beta}}\right)\right)^{\theta}\right]^{\frac{1}{\theta}}\right)}\right\rangle$ \\
\hline & Scalar Multiplication operation & $\left\langle\frac{1-\exp \left(-\left[k\left(-\ln \left(\frac{1-\mu_{\alpha}}{1+\lambda \mu_{\alpha}}\right)\right)^{\theta}\right]^{\frac{1}{\theta}}\right)}{1+\lambda \exp \left(-\left[k\left(-\ln \left(\frac{1-\mu_{\alpha}}{1+\lambda \mu_{\alpha}}\right)\right)^{\theta}\right]^{\frac{1}{\theta}}\right)}, \exp \left(-\left[k\left(-\ln v_{\alpha}\right)^{\theta}\right]^{\frac{1}{\theta}}\right)\right\rangle$ \\
\hline & Power operation & $\left\langle\exp \left(-\left[k\left(-\ln \mu_{\alpha}\right)^{\theta}\right]^{\frac{1}{\theta}}\right), \frac{1-\exp \left(-\left[k\left(-\ln \left(\frac{1-v_{\alpha}}{1+\lambda \lambda \alpha_{\alpha}}\right)\right)^{\theta}\right]^{\frac{1}{\theta}}\right)}{1+\lambda \exp \left(-\left[k\left(-\ln \left(\frac{1-v_{\alpha}}{1+\lambda v_{\alpha}}\right)\right)^{\theta}\right]^{\frac{1}{\theta}}\right)}\right\rangle$ \\
\hline \multirow[t]{4}{*}{ Clayton } & Addition operation & 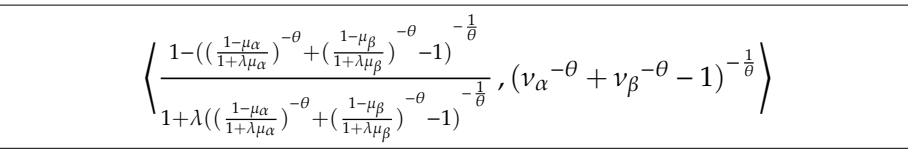 \\
\hline & Multiplication operation & $\left\langle\left(\mu_{\alpha}{ }^{-\theta}+\mu_{\beta}{ }^{-\theta}-1\right)^{-\frac{1}{\theta}}, \frac{1-\left(\left(\frac{1-v_{\alpha}}{1+\lambda v_{\alpha}}\right)^{-\theta}+\left(\frac{1-v_{\beta}}{1+\lambda v_{\beta}}\right)^{-\theta}-1\right)^{-\frac{1}{\theta}}}{1+\lambda\left(\left(\frac{1-v_{\alpha}}{1+\lambda v_{\alpha}}\right)^{-\theta}+\left(\frac{1-v_{\beta}}{1+\lambda v_{\beta}}\right)^{-\theta}-1\right)^{-\frac{1}{\theta}}}\right\rangle$ \\
\hline & Scalar Multiplication operation & $\left\langle\frac{1-\left(k\left(\frac{1-\mu_{\alpha}}{1+\lambda \mu_{\alpha}}\right)^{-\theta}-1\right)^{-\frac{1}{\theta}}}{1+\lambda\left(k\left(\frac{1-\mu_{\alpha}}{1+\lambda \mu_{\alpha}}\right)^{-\theta}-1\right)^{-\frac{1}{\theta}}},\left(k v_{\alpha}^{-\theta}-1\right)^{-\frac{1}{\theta}}\right\rangle$ \\
\hline & Power operation & $\left\langle\left(k \mu_{\alpha}{ }^{-\theta}-1\right)^{-\frac{1}{\theta}}, \frac{1-\left(k\left(\frac{1-v_{\alpha}}{1+\lambda v_{\alpha}}\right)^{-\theta}-1\right)^{-\frac{1}{\theta}}}{1+\lambda\left(k\left(\frac{1-v_{\alpha}}{1+\lambda v_{\alpha}}\right)^{-\theta}-1\right)^{-\frac{1}{\theta}}}\right\rangle$ \\
\hline
\end{tabular}


Table 3. Cont.

\begin{tabular}{|c|c|c|}
\hline Name & Operations & $\begin{array}{r}\text { Formulas } \\
\end{array}$ \\
\hline \multirow[t]{4}{*}{ Ali-Mikhail-Haq } & Addition operation & $\left\langle\frac{1-\frac{\frac{1-\mu_{\alpha}}{1+\lambda \mu_{\alpha}} \frac{1-\mu_{\beta}}{1+\lambda \mu_{\beta}}}{1-\theta\left(1-\frac{1-\mu_{\alpha}}{1+\lambda \mu_{\alpha}}\right)\left(1-\frac{1-\mu_{\beta}}{1+\lambda \mu_{\beta}}\right)}}{1+\lambda\left(\frac{\frac{1-\mu_{\alpha}}{1+\lambda \mu_{\alpha}} \frac{1-\mu_{\beta}}{1+\lambda \mu_{\beta}}}{1-\theta\left(1-\frac{1-\mu_{\alpha}}{1+\lambda \mu_{\alpha}}\right)\left(1-\frac{1-\mu_{\beta}}{1+\lambda \mu_{\beta}}\right)}\right)}, \frac{v_{\alpha} v_{\beta}}{1-\theta\left(1-v_{\alpha}\right)\left(1-v_{\beta}\right)}\right\rangle$ \\
\hline & Multiplication operation & $\left\langle\frac{\mu_{\alpha} \mu_{\beta}}{1-\theta\left(1-\mu_{\alpha}\right)\left(1-\mu_{\beta}\right)}, \frac{1-\frac{\frac{1-v_{\alpha}}{1+\lambda v_{\alpha}} \frac{1-v_{\beta}}{1+\lambda v_{\beta}}}{1-\theta\left(1-\frac{1-v_{\alpha}}{1+\lambda v_{\alpha}}\right)\left(1-\frac{1-v_{\beta}}{1+\lambda v_{\beta}}\right)}}{1+\lambda\left(\frac{\frac{1-\alpha_{\alpha}}{1+\lambda v_{0}}}{1+\lambda v_{\beta}}\right)}\right\rangle$ \\
\hline & Scalar Multiplication operation & $\left\langle\frac{1-\frac{\left(\frac{1-\mu_{\alpha}}{1+\lambda \mu_{\alpha}}{ }^{k}\right.}{1-\theta\left(1-\frac{1-\mu_{\alpha}}{1+\lambda \mu_{\alpha}}\right)^{k}}}{1+\lambda\left(\frac{\left(\frac{1+\mu_{\alpha}}{1+\lambda \mu_{\alpha}}\right)^{k}}{1-\theta\left(1-\frac{1-\mu_{\alpha}}{1+\lambda \mu_{\alpha}}\right)^{k}}\right)}, \frac{v_{\alpha}{ }^{k}}{1-\theta\left(1-v_{\alpha}\right)^{k}}\right)$ \\
\hline & Power operation & $\left\langle\frac{v_{a}{ }^{k}}{1-\theta\left(1-v_{\alpha}\right)^{k}}, \frac{1-\frac{\left(\frac{1-\mu_{\alpha}}{1+\lambda \mu_{\alpha}}\right)^{k}}{1-\theta\left(1-\frac{1-\mu_{\alpha}}{1+\lambda \mu_{\alpha}}\right)^{k}}}{1+\lambda\left(\frac{\left(\frac{1-\mu_{\alpha}}{1+\lambda \mu_{\alpha}}\right)^{k}}{1-\theta\left(1-\frac{1-\mu_{\alpha}}{1+\lambda \mu_{\alpha}}\right)^{k}}\right)}\right\rangle$ \\
\hline \multirow[t]{4}{*}{ Joe } & Addition operation & 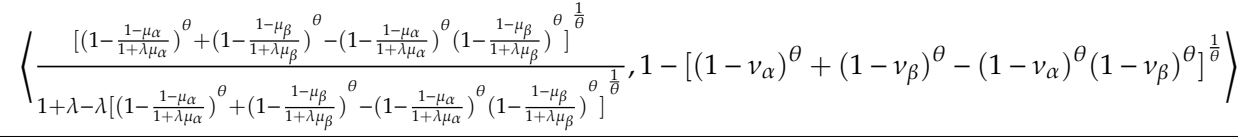 \\
\hline & Multiplication operation & $\left\langle 1-\left[\left(1-\mu_{\alpha}\right)^{\theta}+\left(1-\mu_{\beta}\right)^{\theta}-\left(1-\mu_{\alpha}\right)^{\theta}\left(1-\mu_{\beta}\right)^{\theta}\right]^{\frac{1}{\theta}}, \frac{\left[\left(1-\frac{1-v_{\alpha}}{1+\lambda v_{\alpha}}\right)^{\theta}+\left(1-\frac{1-v_{\beta}}{1+\lambda v_{\beta}}\right)^{\theta}-\left(1-\frac{1-v_{\alpha}}{1+\lambda v_{\alpha}}\right)^{\theta}\left(1-\frac{1-v_{\beta}}{1+\lambda v_{\beta}}\right)^{\theta}\right]^{\frac{1}{\theta}}}{1+\lambda-\lambda\left[\left(1-\frac{1-v_{\alpha}}{1+\lambda \nu v_{\alpha}}\right)^{\theta}+\left(1-\frac{1-v_{\beta}}{1+\lambda v_{\beta}}\right)^{\theta}-\left(1-\frac{1-v_{\alpha}}{1+\lambda v_{\alpha}}\right)^{\theta}\left(1-\frac{1-v_{\beta}}{1+\lambda v_{\beta}}\right)^{\theta \frac{1}{\theta}}\right.}\right\rangle$ \\
\hline & Scalar Multiplication operation & 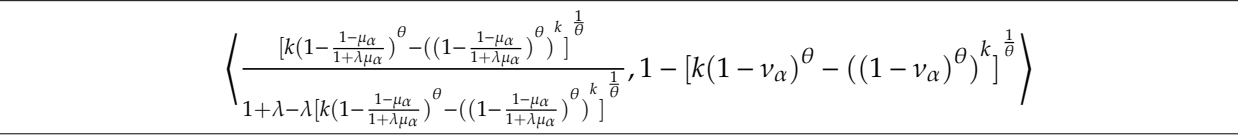 \\
\hline & Power operation & $\left\langle 1-\left[k\left(1-\mu_{\alpha}\right)^{\theta}-\left(\left(1-\mu_{\alpha}\right)^{\theta}\right)^{k}\right]^{\frac{1}{\theta}}, \frac{\left[k\left(1-\frac{1-v_{\alpha}}{1+\lambda v_{\alpha}}\right)^{\theta}-\left(\left(1-\frac{1-v_{\alpha}}{1+\nu_{\alpha}}\right)^{\theta}\right)^{k}\right]^{\frac{1}{\theta}}}{1+\lambda-\lambda\left[k\left(1-\frac{1-v_{\alpha}}{1+\lambda v_{\alpha}}\right)^{\theta}-\left(\left(1-\frac{1-v_{\alpha}}{1+\lambda v_{\alpha}}\right)^{\theta}\right)^{k^{\frac{1}{\theta}}}\right]^{\frac{1}{\theta}}}\right\rangle$ \\
\hline
\end{tabular}


In the following Section, we propose the development of intuitionistic fuzzy Archimedean copula weighted arithmetic and geometric aggregation operators, which is achieved. Furthermore, the properties of those extended aggregation operators are investigated.

\section{Novel Intuitionistic Fuzzy Archimedean Copula Weighted Arithmetic and Geometric Aggregation Operators}

\subsection{Intuitionistic Fuzzy Archimedean Copula Weighted Arithmetic Aggregation Operator}

Definition 7. Let $\alpha_{i}=\left\langle\mu_{\alpha_{i}}, v_{\alpha_{i}}\right\rangle, i=1, \ldots, n$, be a collection of IFVs and $w=\left\{w_{1}, \ldots, w_{n}\right\}^{T}$ be the weighting vector of $\alpha_{i}$, with $0 \leq w_{i} \leq 1$ and $\sum_{i=1}^{n} w_{i}=1$. An intuitionistic fuzzy Archimedean copula weighted arithmetic aggregation (IFACWA $A_{w}$ ) operator of dimension $n$ is a mapping, IFACWA $A_{w}: V^{n} \rightarrow V$, according to: $\operatorname{IFACWAA}_{w}\left(\alpha_{1}, \ldots, \alpha_{n}\right)=\oplus_{C_{i=1}^{n}}^{n} w_{i} \alpha_{i}$.

Based on the proposed addition and scalar multiplication operations of IFVs, the following theorem is expressed.

Theorem 3. Let $\alpha_{i}=\left\langle\mu_{\alpha_{i}}, v_{\alpha_{i}}\right\rangle, i=1, \ldots, n$, be a collection of IFVs and $w=\left\{w_{1}, \ldots, w_{n}\right\}^{T}$ be the associated weighting vector, with $0 \leq w_{i} \leq 1$ and $\sum_{i=1}^{n} w_{i}=1$. Then, the aggregated value, based on the IFACWAA $A_{w}$ operator, can be expressed as:

$$
\operatorname{IFACWA} A_{w}\left(\alpha_{1}, \ldots, \alpha_{n}\right)=\left\langle n_{\lambda}\left(\varphi^{-1}\left[\sum_{i=1}^{n} w_{i} \varphi\left(n_{\lambda}\left(\mu_{\alpha_{i}}\right)\right)\right]\right), \varphi^{-1}\left[\sum_{i=1}^{n} w_{i} \varphi\left(v_{\alpha_{i}}\right)\right]\right\rangle
$$

The value of IFACWAA $A_{w}$ operator is also an IFV.

Proof of Theorem 3. For $n=1$, we get:

$$
\operatorname{IFACWA} A_{w}\left(\alpha_{1}\right)=\left\langle n_{\lambda}\left(\varphi^{-1}\left[w_{1} \varphi\left(n_{\lambda}\left(\mu_{\alpha_{1}}\right)\right)\right]\right), \varphi^{-1}\left[w_{1} \varphi\left(v_{\alpha_{1}}\right)\right]\right\rangle
$$

But we have that $w_{1}=1$, so we get:

$$
\begin{gathered}
\operatorname{IFACWAA_{w}}\left(\alpha_{1}\right)=\left\langle n_{\lambda}\left(\varphi^{-1}\left[\varphi\left(n_{\lambda}\left(\mu_{\alpha_{1}}\right)\right)\right]\right), \varphi^{-1}\left[\varphi\left(v_{\alpha_{1}}\right)\right]\right\rangle \Leftrightarrow \\
\operatorname{IFACWAA}_{w}\left(\alpha_{1}\right)=\left\langle\mu_{\alpha_{1}}, v_{\alpha_{1}}\right\rangle \Leftrightarrow \operatorname{IFACWAA_{w}}\left(\alpha_{1}\right)=\alpha_{1},
\end{gathered}
$$

as $\alpha_{1} \in V, I F A C W A A_{w}\left(\alpha_{1}\right) \in V$.

Next, we suppose that for $n=k$ it holds, so we calculate:

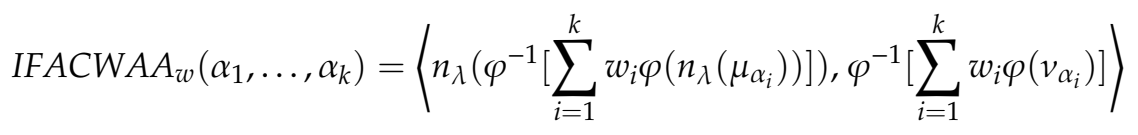

For $n=k+1$, we get:

$$
\begin{aligned}
& \operatorname{IFACWAA_{w}}\left(\alpha_{1}, \ldots, \alpha_{k}, \alpha_{k+1}\right)=\oplus_{C_{i=1}^{k+1}}^{k+1} w_{i} \alpha_{i}=\left(\oplus_{C_{i=1}^{k}}^{k} w_{i} \alpha_{i}\right) \oplus_{C}\left(w_{k+1} \alpha_{k+1}\right) \\
& =\left\langle n_{\lambda}\left(\varphi^{-1}\left[\sum_{i=1}^{k} w_{i} \varphi\left(n_{\lambda}\left(\mu_{\alpha_{i}}\right)\right)\right]\right), \varphi^{-1}\left[\sum_{i=1}^{k} w_{i} \varphi\left(v_{\alpha_{i}}\right)\right]\right\rangle \oplus_{C}\left\langle n_{\lambda}\left(\varphi^{-1}\left[w_{k+1} \varphi\left(n_{\lambda}\left(\mu_{\alpha_{k+1}}\right)\right)\right]\right), \varphi^{-1}\left[w_{k+1} \varphi\left(v_{\alpha_{k+1}}\right)\right]\right\rangle \\
& =\left\langle n_{\lambda}\left(\varphi^{-1}\left[\varphi\left(n_{\lambda}\left(n_{\lambda}\left(\varphi^{-1}\left[\sum_{i=1}^{k} w_{i} \varphi\left(n_{\lambda}\left(\mu_{\alpha_{i}}\right)\right)\right]\right)\right)\right)+\varphi\left(n_{\lambda}\left(n_{\lambda}\left(\varphi^{-1}\left[w_{k+1} \varphi\left(n_{\lambda}\left(\mu_{\alpha_{k+1}}\right)\right)\right]\right)\right)\right)\right]\right),\right.
\end{aligned}
$$




$$
\begin{gathered}
\left.\varphi^{-1}\left[\varphi\left(\varphi^{-1}\left[\sum_{i=1}^{k} w_{i} \varphi\left(v_{\alpha_{i}}\right)\right]\right)+\varphi\left(\varphi^{-1}\left[w_{k+1} \varphi\left(v_{\alpha_{k+1}}\right)\right]\right)\right]\right\rangle=\left\langle n_{\lambda}\left(\varphi^{-1}\left[\sum_{i=1}^{k} w_{i} \varphi\left(n_{\lambda}\left(\mu_{\alpha_{i}}\right)\right)+w_{k+1} \varphi\left(n_{\lambda}\left(\mu_{\alpha_{k+1}}\right)\right)\right]\right),\right. \\
\left.\left.\left.\varphi^{-1}\left[\sum_{i=1}^{k} w_{i} \varphi\left(v_{\alpha_{i}}\right)\right]\right)+w_{k+1} \varphi\left(v_{\alpha_{k+1}}\right)\right]\right\rangle=\left\langle n_{\lambda}\left(\varphi^{-1}\left[\sum_{i=1}^{k+1} w_{i} \varphi\left(n_{\lambda}\left(\mu_{\alpha_{i}}\right)\right)\right]\right), \varphi^{-1}\left[\sum_{i=1}^{k+1} w_{i} \varphi\left(v_{\alpha_{i}}\right)\right]\right\rangle
\end{gathered}
$$

It holds, so as a result, by mathematical induction the proof is complete.

Proposition 4. Let $\alpha_{i}=\left\langle\mu_{\alpha_{i}}, v_{\alpha_{i}}\right\rangle, i=1, \ldots, n$, be a collection of IFVs and $w=\left\{w_{1}, \ldots, w_{n}\right\}^{T}$ be the associated weighting vector, with $0 \leq w_{i} \leq 1$ and $\sum_{i=1}^{n} w_{i}=1$. Then IFACWA $A_{w}$ operator is:

1. Idempotent, i.e., if $\left\langle\mu_{\alpha_{i}}, v_{\alpha_{i}}\right\rangle=\left\langle\mu_{\alpha}, v_{\alpha}\right\rangle$, then: for $i=1, \ldots, n$, IFACWA $A_{w}\left(\alpha_{1}, \ldots, \alpha_{n}\right)=\alpha$.

2. Bounded, i.e., for $i=1, \ldots, n,\langle 0,1\rangle \leq \operatorname{IFACWAA_{w}}\left(\alpha_{1}, \ldots, \alpha_{n}\right) \leq\langle 1,0\rangle$.

3. Commutative, i.e., for any permutation of $\alpha_{i}=\left\langle\mu_{\alpha_{i}}, v_{\alpha_{i}}\right\rangle, \alpha_{f(i)}=\left\langle\mu_{\alpha_{f(i)}}, v_{\alpha_{f(i)}}\right\rangle$, with $i=1, \ldots, n$, where $f:\{1,2, \ldots, n\} \rightarrow\{1,2, \ldots, n\}, \operatorname{IFACWAA}_{w}\left(\alpha_{1}, \ldots, \alpha_{n}\right)=\operatorname{IFACWAA}_{w}\left(\alpha_{f(1)}, \ldots, \alpha_{f(n)}\right)$.

Proof of Proposition 4. 1. IFACWA $A_{w}\left(\alpha_{1}, \ldots, \alpha_{n}\right)=\left\langle n_{\lambda}\left(\varphi^{-1}\left[\sum_{i=1}^{n} w_{i} \varphi\left(n_{\lambda}\left(\mu_{\alpha_{i}}\right)\right)\right]\right), \varphi^{-1}\left[\sum_{i=1}^{n} w_{i} \varphi\left(v_{\alpha_{i}}\right)\right]\right\rangle$. As $\mu_{\alpha_{i}}=\mu_{\alpha}$ and $v_{\alpha_{i}}=v_{\alpha}$ we get: IFACWAA $A_{w}\left(\alpha_{1}, \ldots, \alpha_{n}\right)=$ $\left\langle n_{\lambda}\left(\varphi^{-1}\left[\sum_{i=1}^{n} w_{i} \varphi\left(n_{\lambda}\left(\mu_{\alpha}\right)\right)\right]\right), \varphi^{-1}\left[\sum_{i=1}^{n} w_{i} \varphi\left(v_{\alpha}\right)\right]\right\rangle=\quad\left\langle n_{\lambda}\left(\varphi^{-1}\left[\varphi\left(n_{\lambda}\left(\mu_{\alpha}\right)\right) \sum_{i=1}^{n} w_{i}\right]\right), \varphi^{-1}\left[\varphi\left(v_{\alpha}\right) \sum_{i=1}^{n} w_{i}\right]\right\rangle=$ $\left\langle n_{\lambda}\left(\varphi^{-1}\left[\varphi\left(n_{\lambda}\left(\mu_{\alpha}\right)\right)\right]\right), \varphi^{-1}\left[\varphi\left(v_{\alpha}\right)\right]\right\rangle=\left\langle\mu_{\alpha}, v_{\alpha}\right\rangle=\alpha$.

The proofs of Equations (2) and (3) are obvious based on the properties of Archimedean copulas and their corresponding co-copulas.

\subsection{Intuitionistic Fuzzy Archimedean Copula Weighted Geometric Aggregation Operator}

Definition 8. Let $\alpha_{i}=\left\langle\mu_{\alpha_{i}}, v_{\alpha_{i}}\right\rangle, i=1, \ldots, n$, be a collection of IFVs and $w=\left\{w_{1}, \ldots, w_{n}\right\}^{T}$ be the weighting vector of $\alpha_{i}$, with $0 \leq w_{i} \leq 1$ and $\sum_{i=1}^{n} w_{i}=1$. An intuitionistic fuzzy Archimedean copula weighted geometric aggregation (IFACWGA $A_{w}$ ) operator of dimension $n$ is a mapping, IFACWGA $A_{w}: V^{n} \rightarrow V$, according to: IFACWGA $A_{w}\left(\alpha_{1}, \ldots, \alpha_{n}\right)=\otimes_{C_{i=1}^{n}}^{n} \alpha_{i}^{w_{i}}$.

Based on the proposed multiplication and power operations of IFVs, the following theorem is expressed.

Theorem 5. Let $\alpha_{i}=\left\langle\mu_{\alpha_{i}}, v_{\alpha_{i}}\right\rangle, i=1, \ldots, n$, be a collection of IFVs and $w=\left\{w_{1}, \ldots, w_{n}\right\}^{T}$ be the associated weighting vector, with $0 \leq w_{i} \leq 1$ and $\sum_{i=1}^{n} w_{i}=1$. Then, the aggregated value, based on the IFACWGA operator, can be expressed as: IFACWGA $A_{w}\left(\alpha_{1}, \ldots, \alpha_{n}\right)=\left\langle\varphi^{-1}\left[\sum_{i=1}^{n} w_{i} \varphi\left(\mu_{\alpha_{i}}\right)\right], n_{\lambda}\left(\varphi^{-1}\left[\sum_{i=1}^{n} w_{i} \varphi\left(n_{\lambda}\left(v_{\alpha_{i}}\right)\right)\right]\right)\right\rangle$.

The value of IFACWGA $A_{w}$ operator is also an IFV.

Proof of Theorem 5. For $n=1$, we get: IFACWGA $A_{w}\left(\alpha_{1}\right)=\left\langle\varphi^{-1}\left[w_{1} \varphi\left(\mu_{\alpha_{1}}\right)\right], n_{\lambda}\left(\varphi^{-1}\left[w_{1} \varphi\left(n_{\lambda}\left(v_{\alpha_{1}}\right)\right)\right]\right)\right\rangle$, but we have that $w_{1}=1$ so we get: IFACWGA $A_{w}\left(\alpha_{1}\right)=\left\langle\varphi^{-1}\left[\varphi\left(\mu_{\alpha_{1}}\right)\right], n_{\lambda}\left(\varphi^{-1}\left[\varphi\left(n_{\lambda}\left(v_{\alpha_{1}}\right)\right)\right]\right)\right\rangle$. As a result, we have: IFACWGA $A_{w}\left(\alpha_{1}\right)=\left\langle\mu_{\alpha_{1}}, v_{\alpha_{1}}\right\rangle=\alpha_{1}$. As $\alpha_{1} \in V$,IFACWGA $A_{w}\left(\alpha_{1}\right) \in V$.

Next, suppose that for $n=k$ it holds.

For $n=k+1$ we calculate:

$$
\begin{gathered}
\operatorname{IFACWGA} A_{w}\left(\alpha_{1}, \ldots, \alpha_{k+1}\right)=\otimes_{C_{i=1}^{k+1}}^{k+1} \alpha_{i}^{w_{i}}=\left(\otimes_{C_{i=1}^{k}}^{k} \alpha_{i}^{w_{i}}\right) \otimes_{C}\left(\alpha_{k+1} w_{k+1}\right) \\
=\left\langle\varphi^{-1}\left[\sum_{i=1}^{k} w_{i} \varphi\left(\mu_{\alpha_{i}}\right)\right], n_{\lambda}\left(\varphi^{-1}\left[\sum_{i=1}^{k} w_{i} \varphi\left(n_{\lambda}\left(v_{\alpha_{i}}\right)\right)\right]\right)\right\rangle \otimes_{\mathcal{C}}\left\langle\varphi^{-1}\left[w_{k+1} \varphi\left(\mu_{\alpha_{k+1}}\right)\right], n_{\lambda}\left(\varphi^{-1}\left[w_{k+1} \varphi\left(n_{\lambda}\left(v_{\alpha_{k+1}}\right)\right)\right]\right)\right\rangle
\end{gathered}
$$




$$
\begin{gathered}
=\left\langle\varphi^{-1}\left[\varphi\left(\varphi^{-1}\left[\sum_{i=1}^{k} w_{i} \varphi\left(\mu_{\alpha_{i}}\right)\right]\right)+\varphi\left(\varphi^{-1}\left[w_{k+1} \varphi\left(\mu_{\alpha_{k+1}}\right)\right]\right)\right], n_{\lambda}\left(\varphi ^ { - 1 } \left[\varphi\left(n_{\lambda}\left(n_{\lambda}\left(\varphi^{-1}\left[\sum_{i=1}^{k} w_{i} \varphi\left(n_{\lambda}\left(v_{\alpha_{i}}\right)\right)\right]\right)\right)\right)\right.\right.\right. \\
\left.\left.\left.+\varphi\left(n_{\lambda}\left(n_{\lambda}\left(\varphi^{-1}\left[w_{k+1} \varphi\left(n_{\lambda}\left(v_{\alpha_{k+1}}\right)\right]\right)\right)\right)\right]\right]\right)\right\rangle=\left\langle\varphi^{-1}\left[\sum_{i=1}^{k} w_{i} \varphi\left(\mu_{\alpha_{i}}\right)+w_{k+1} \varphi\left(\mu_{\alpha_{k+1}}\right)\right], n_{\lambda}\left(\varphi^{-1}\left[\sum_{i=1}^{k} w_{i} \varphi\left(n_{\lambda}\left(v_{\alpha_{i}}\right)\right)+w_{k+1} \varphi\left(n_{\lambda}\left(v_{\alpha_{k+1}}\right)\right)\right]\right)\right\rangle \\
=\left\langle\varphi^{-1}\left[\sum_{i=1}^{k+1} w_{i} \varphi\left(\mu_{\alpha_{i}}\right)\right], n_{\lambda}\left(\varphi^{-1}\left[\sum_{i=1}^{k+1} w_{i} \varphi\left(n_{\lambda}\left(v_{\alpha_{i}}\right)\right)\right]\right)\right\rangle
\end{gathered}
$$

It holds, so by mathematical induction the proof is complete. $\square$

Remark 1. IFACWGA $A_{w}$ operator possesses the same properties as IFACWA $A_{w}$, such as idempotency, boundary property and commutativity.

In order to depict some of the produced IFACWAA $A_{w}$ and IFACWGA $A_{w}$ operators we use the proposed operators for IFVs as described in Table 3, which are expressed in the Tables 4 and 5, respectively.

\begin{tabular}{|c|c|}
\hline Name & IFACWAA $_{w}$ \\
\hline Gumbel & $\left\langle\frac{1-\exp \left[\left[-\sum_{i=1}^{n} w_{i}\left[-\ln \left(\frac{1-\mu \alpha_{i}}{1+\lambda \mu \alpha_{i}}\right)\right]^{\theta}\right]^{\frac{1}{\theta}}\right]}{1+\lambda\left(\exp \left[\left[-\sum_{i=1}^{n} w_{i}\left[-\ln \left(\frac{1-\mu_{\alpha_{i}}}{1+\lambda \mu \alpha_{i}}\right)\right]^{\theta}\right]^{\frac{1}{\theta}}\right]\right)}, \exp \left[\left[-\sum_{i=1}^{n} w_{i}\left[-\ln v_{\alpha_{i}}\right]^{\theta}\right]^{\frac{1}{\theta}}\right]\right\rangle$ \\
\hline Clayton & $\left\langle\frac{1-\left(\sum_{i=1}^{n} w_{i}\left(\frac{1-\mu \alpha_{i}}{1+\lambda \mu \alpha_{i}}\right)^{-\theta}\right)^{-\frac{1}{\theta}}}{1+\lambda\left(\left(\sum_{i=1}^{n} w_{i}\left(\frac{1-\mu \alpha_{i}}{1+\lambda \mu \alpha_{i}}\right)^{-\theta}\right)^{-\frac{1}{\theta}}\right)},\left(\sum_{i=1}^{n} w_{i}\left(v_{\alpha_{i}}\right)^{-\theta}\right)^{-\frac{1}{\theta}}\right\rangle$ \\
\hline Ali-Mikhail-Haq & 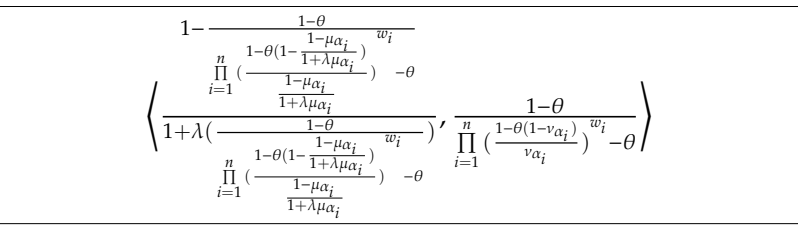 \\
\hline Joe & $\left\langle\frac{\left(1-\prod_{i=1}^{n}\left(1-\left(1-\frac{1-\mu \alpha_{\alpha_{i}}}{1+\lambda \mu \alpha_{i}}\right)^{\theta}\right)^{v_{i}}\right)^{1 / \theta}}{1+\lambda\left(1-\left(1-\prod_{i=1}^{n}\left(1-\left(1-\frac{1-\mu \alpha_{\alpha_{i}}}{1+\lambda \mu \alpha_{i}}\right)^{\theta}\right)^{w_{i}}\right)^{1 / \theta}\right)}, 1-\left(1-\prod_{i=1}^{n}\left(1-\left(1-v_{\alpha_{i}}\right)^{\theta}\right)^{w_{i}}\right)^{1 / \theta}\right\rangle$ \\
\hline
\end{tabular}

Table 4. Some Sugeno-Based Intuitionistic Fuzzy Archimedean Copula Weighted Arithmetic Aggregation Operators.

\begin{tabular}{|c|c|}
\hline Name & IFACWGA $A_{w}$ \\
\hline Gumbel & $\left\langle\exp \left[\left[-\sum_{i=1}^{n} w_{i}\left[-\ln \mu_{\alpha_{i}}\right]^{\theta}\right]^{\frac{1}{\theta}}\right], \frac{1-\exp \left[\left[-\sum_{i=1}^{n} w_{i}\left[-\ln \left(\frac{1-v_{\alpha_{i}}}{1+\lambda v_{\alpha_{i}}}\right)\right]^{\theta}\right]^{\frac{1}{\theta}}\right]}{1+\lambda\left(\exp \left[\left[-\sum_{i=1}^{n} w_{i}\left[-\ln \left(\frac{1-v_{\alpha_{i}}}{1+\lambda v_{\alpha_{i}}}\right)\right]^{\theta}\right]^{\frac{1}{\theta}}\right]\right)}\right\rangle$ \\
\hline Clayton & $\left\langle\left(\sum_{i=1}^{n} w_{i}\left(\mu_{\alpha_{i}}\right)^{-\theta}\right)^{-\frac{1}{\theta}}, \frac{1-\left(\sum_{i=1}^{n} w_{i}\left(\frac{1-v_{\alpha_{i}}}{1+\lambda v_{\alpha_{i}}}\right)^{-\theta}\right)^{-\frac{1}{\theta}}}{1+\lambda\left(\left(\sum_{i=1}^{n} w_{i}\left(\frac{1-v_{\alpha_{i}}}{1+\lambda v_{\alpha_{i}}}\right)^{-\theta}\right)^{-\frac{1}{\theta}}\right.}\right\rangle$ \\
\hline Ali-Mikhail-Haq & 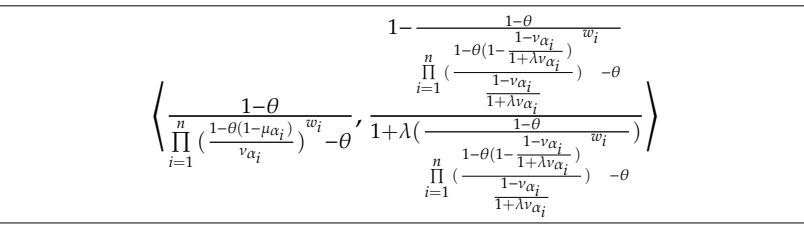 \\
\hline Joe & $\left\langle 1-\left(1-\prod_{i=1}^{n}\left(1-\left(1-\mu_{\alpha_{i}}\right)^{\theta}\right)^{w_{i}}\right)^{1 / \theta}, \frac{\left(1-\prod_{i=1}^{n}\left(1-\left(1-\frac{1-v_{\alpha_{i}}}{1+\lambda v_{\alpha_{i}}}\right)^{\theta}\right)^{w_{i}}\right)^{1 / \theta}}{1+\lambda\left(1-\left(1-\prod_{i=1}^{n}\left(1-\left(1-\frac{1-v_{\alpha_{i}}}{1+\lambda v_{\alpha_{i}}}\right)^{\theta}\right)^{w_{i}}\right)^{1 / \theta}\right)}\right\rangle$ \\
\hline
\end{tabular}

Table 5. Some Sugeno-Based Intuitionistic Fuzzy Archimedean Copula Weighted Geometric Aggregation Operators. 


\section{IFACWAA $A_{w}$ and IFACWGA $A_{w}$ Operators in MADM}

In this section an algorithm for Multiple-Attribute Decision Making (MADM) with IFVs is introduced. Let $U=\left\{u_{1}, \ldots, u_{m}\right\}$ be the set of alternatives and $C=\left\{C_{1}, \ldots, C_{n}\right\}$ be the set of attributes, with a weighting vector $w=\left\{w_{1}, \ldots, w_{n}\right\}^{T}$, with $0 \leq w_{i} \leq 1$ and $\sum_{i=1}^{n} w_{i}=1$. The intuitionistic fuzzy decision matrix $R=\left(\left\langle\mu_{i j}, v_{i j}\right\rangle\right)_{m \times n}$ where $\alpha_{i j}=\left\langle\mu_{i j}, v_{i j}\right\rangle$, with $i=1, \ldots, m$ and $j=1, \ldots, n$ represent the degree that $u_{i}$ satisfies $C_{j}$, such that $\mu_{i j}, v_{i j} \in[0,1]$, with $\mu_{i j}+v_{i j} \leq 1$, i.e.,

$$
R=\left[\begin{array}{ccc}
<\mu_{11}, v_{11}> & \cdots & <\mu_{1 n}, v_{1 n}> \\
\vdots & \ddots & \vdots \\
<\mu_{m 1}, v_{m 1}> & \cdots & <\mu_{m n}, v_{m n}>
\end{array}\right]
$$

In order to obtain the weighting vector of attributes, the modified maximizing deviations method for MADM with intuitionistic fuzzy information [9] can be used. As MADM with intuitionistic fuzzy information is a procedure to end up with the best alternative $u_{i} \in U, i=1, \ldots, m$, the comparison of IFVs is achieved by the following concept.

For any IFV $\alpha=\langle\mu, v\rangle$, Chen and Tan [33] proposed a score function $s$ and Hong and Choi [34] proposed an accuracy function $h$, i.e., $s(\alpha)=\mu_{\alpha}-v_{\alpha}$ and $h(\alpha)=\mu_{\alpha}+v_{\alpha}$, respectively. The larger the score is, the greater the IFV would be. In the case that the score function's values for two IFVs are equal; the accuracy function can provide more specific result. Xu and Yager [35], based on score and accuracy functions, gave a total order for IFVs.

Based on the above and inspired by MADM model in [9], we derive the following algorithm.

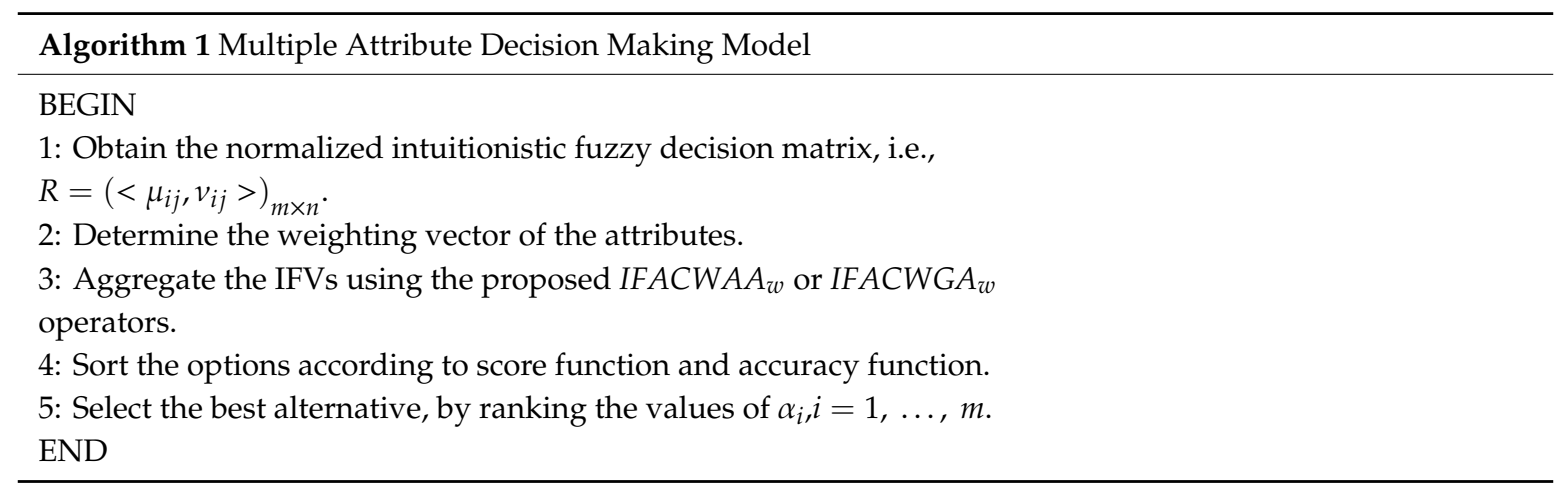

In order to demonstrate the proposed method, we provide the following numerical example, which is adapted from Tao et al.'s [9] work, in order to compare their method with the present extension of it.

\section{A Practical Example for MADM with IFVs.}

A research facility has arranged to purchase an electronic device. The people in charge, selected the four most appropriate models for further consideration, after the market research. Those are indicated as the four alternatives $U=\left\{u_{1}, u_{2}, u_{3}, u_{4}\right\}$. The evaluation of the alternatives is achieved by the consideration of five attributes by the decision maker (DM). Those attributes are listed as follows: the layout of the product $\left(C_{1}\right)$, the technical assistance $\left(C_{2}\right)$, the brand $\left(C_{3}\right)$, the price $\left(C_{4}\right)$ and the quality of the product $\left(C_{5}\right)$. 
Step 1. The normalized intuitionistic fuzzy decision matrix can be obtained as follows:

$$
R=\left(\alpha_{i j}\right)_{4 \times 5}=\left[\begin{array}{ccccc}
\langle 0.4,0.3\rangle & \langle 0.5,0.2\rangle & \langle 0.7,0.2\rangle & \langle 0.4,0.6\rangle & \langle 0.6,0.2\rangle \\
\langle 0.6,0.1\rangle & \langle 0.4,0.3\rangle & \langle 0.3,0.5\rangle & \langle 0.6,0.2\rangle & \langle 0.5,0.3\rangle \\
\langle 0.5,0.4\rangle & \langle 0.6,0.1\rangle & \langle 0.6,0.2\rangle & \langle 0.7,0.1\rangle & \langle 0.3,0.6\rangle \\
\langle 0.6,0.3\rangle & \langle 0.4,0.5\rangle & \langle 0.5,0.3\rangle & \langle 0.8,0.2\rangle & \langle 0.5,0.2\rangle
\end{array}\right] .
$$

in which, the element $\left\langle\mu_{i j}, v_{i j}\right\rangle$, with $i=1, \ldots, 4$ and $j=1, \ldots, 5$, represents the intuitionistic membership and non-membership degree of the $i$-th alternative that satisfies the $j$-th attribute.

Step 2. In the present work, we use Tao et al.'s [9], modified maximizing deviations method, in order to derive the five weightings of the attributes, and since the numerical example is the same we get: $w_{1}=0.2162, w_{2}=0.2703, w_{3}=0.1892, w_{4}=0.1081, w_{5}=0.2162$.

Step 3. The aggregated results are given by the usage of IFACWAA $A_{w}$ and IFACWGA $A_{w}$ operators and are listed in Tables 6 and 7.

Step 4. The score function's results for each alternative and for each type of IFACWAA IFACWGA $A_{w}$ operators are listed in Tables 8 and 9.

Step 5. The ranking of the alternatives is achieved and is depicted in Table 10.

Table 6. The aggregation results using intuitionistic fuzzy Archimedean copula weighted arithmetic aggregation (IFACWAA $\left.A_{w}\right)$

\begin{tabular}{ccccc}
\hline & Gumbel & Clayton & Ali-Mikhail-Haq & Joe \\
Alternatives & $\boldsymbol{\theta}=1$ & $\boldsymbol{\theta}=1$ & $\boldsymbol{\theta}=-1$ & $\boldsymbol{\lambda}$ \\
& $\lambda=0.3$ & $\lambda=0.3$ & $\lambda=0.3$ & $\lambda=0.3$ \\
\hline$u_{1}$ & $\langle 0.5394,0.2459\rangle$ & $\langle 0.5560,0.2337\rangle$ & $\langle 0.5356,0.2488\rangle$ & $\langle 0.5394,0.2459\rangle$ \\
$u_{2}$ & $\langle 0.4777,0.2494\rangle$ & $\langle 0.4908,0.2126\rangle$ & $\langle 0.4736,0.2544\rangle$ & $\langle 0.4777,0.2494\rangle$ \\
$u_{3}$ & $\langle 0.5388,0.2267\rangle$ & $\langle 0.5556,0.1776\rangle$ & $\langle 0.5338,0.2357\rangle$ & $\langle 0.5388,0.2267\rangle$ \\
$u_{4}$ & $\langle 0.5444,0.3020\rangle$ & $\langle 0.5731,0.2846\rangle$ & $\langle 0.5395,0.3056\rangle$ & $\langle 0.5444,0.3020\rangle$ \\
\hline
\end{tabular}

Table 7. The aggregation results using intuitionistic fuzzy Archimedean copula weighted geometric aggregation (IFACWGA $\left.A_{w}\right)$

\begin{tabular}{ccccc}
\hline & Gumbel & Clayton & Ali-Mikhail-Haq & Joe \\
Alternatives & $\boldsymbol{\theta}=1$ & $\boldsymbol{\theta}=1$ & $\boldsymbol{\theta}=-1$ & $\lambda=0.3$ \\
& $\lambda=0.3$ & $\lambda=0.3$ & $\lambda=0.3$ \\
\hline$u_{1}$ & $\langle 0.5156,0.2762\rangle$ & $\langle 0.5045,0.2976\rangle$ & $\langle 0.5197,0.2693\rangle$ & $\langle 0.5156,0.2762\rangle$ \\
$u_{2}$ & $\langle 0.4534,0.2940\rangle$ & $\langle 0.4387,0.3097\rangle$ & $\langle 0.4576,0.2868\rangle$ & $\langle 0.4534,0.2940\rangle$ \\
$u_{3}$ & $\langle 0.5049,0.3176\rangle$ & $\langle 0.4823,0.3581\rangle$ & $\langle 0.5113,0.3012\rangle$ & $\langle 0.5049,0.3176\rangle$ \\
$u_{4}$ & $\langle 0.5152,0.3306\rangle$ & $\langle 0.5045,0.3443\rangle$ & $\langle 0.5196,0.3252\rangle$ & $\langle 0.5152,0.3306\rangle$ \\
\hline
\end{tabular}

Table 8. The score function's results using IFACWAA $A_{w}$.

\begin{tabular}{ccccc}
\hline & Gumbel & Clayton & Ali-Mikhail-Haq & Joe \\
Alternatives & $\boldsymbol{\theta}=1$ & $\boldsymbol{\theta}=1$ & $\boldsymbol{\theta}=-1$ & $\lambda=1$ \\
& $\lambda=0.3$ & $\lambda=0.3$ & $0.3=0.3$ & 0.2935 \\
\hline$u_{1}$ & 0.2935 & 0.3223 & 0.2868 & 0.2283 \\
$u_{2}$ & 0.2283 & 0.2782 & 0.2192 & 0.3121 \\
$u_{3}$ & 0.3121 & 0.3780 & 0.2981 & 0.2424 \\
\hline
\end{tabular}


Table 9. The score function's results using IFACWGA $A_{w}$

\begin{tabular}{ccccc}
\hline & Gumbel & Clayton & Ali-Mikhail-Haq & Joe \\
Alternatives & $\boldsymbol{\theta}=1$ & $\boldsymbol{\theta}=1$ & $\boldsymbol{\theta}=-1$ & $\boldsymbol{\theta}=1$ \\
& $\lambda=0.3$ & $\lambda=0.3$ & 0.2504 & $\lambda=0.3$ \\
\hline$u_{1}$ & 0.2394 & 0.2069 & 0.1708 & 0.2394 \\
$u_{2}$ & 0.1594 & 0.1290 & 0.2101 & 0.1594 \\
$u_{3}$ & 0.1873 & 0.1243 & 0.1944 & 0.1873 \\
$u_{4}$ & 0.1846 & 0.1602 & 0.1846 \\
\hline
\end{tabular}

Table 10. Ranking of the alternatives.

\begin{tabular}{ccc}
\hline Type & IFACWAA $_{\boldsymbol{w}}$ & $\boldsymbol{I F A C W G A}_{\boldsymbol{w}}$ \\
\hline Gumbel & $u_{3}>u_{1}>u_{4}>u_{2}$ & $u_{1}>u_{3}>u_{4}>u_{2}$ \\
Clayton & $u_{3}>u_{1}>u_{4}>u_{2}$ & $u_{1}>u_{4}>u_{2}>u_{3}$ \\
Ali-Mikhail-Haq & $u_{3}>u_{1}>u_{4}>u_{2}$ & $u_{1}>u_{3}>u_{4}>u_{2}$ \\
Joe & $u_{3}>u_{1}>u_{4}>u_{2}$ & $u_{1}>u_{3}>u_{4}>u_{2}$ \\
\hline
\end{tabular}

To illustrate the affection of $\lambda$ parameter in the procedure as $\theta$ parameter is fixed to 1 (and -1 in Ali-Mikhail-Haq case) we utilized the following graphs that are depicted in Figures 1 and 2 for the IFACWA $A_{w}$ and in Figures 3 and 4 for the IFACWGA $A_{w}$

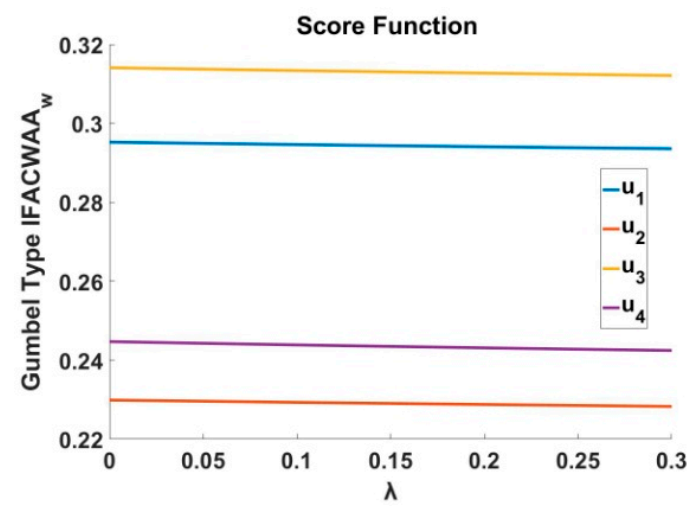

(A)

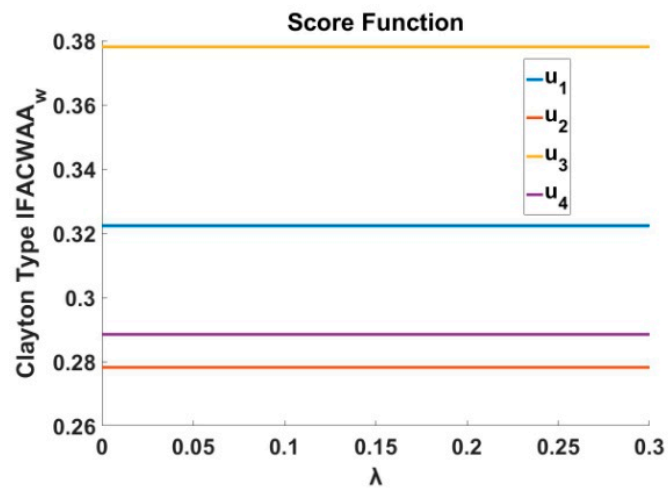

(B)

Figure 1. (A) Gumbel-type IFACWAA $A_{w}$ score result. (B) Clayton-type IFACWAA $A_{w}$ score result.

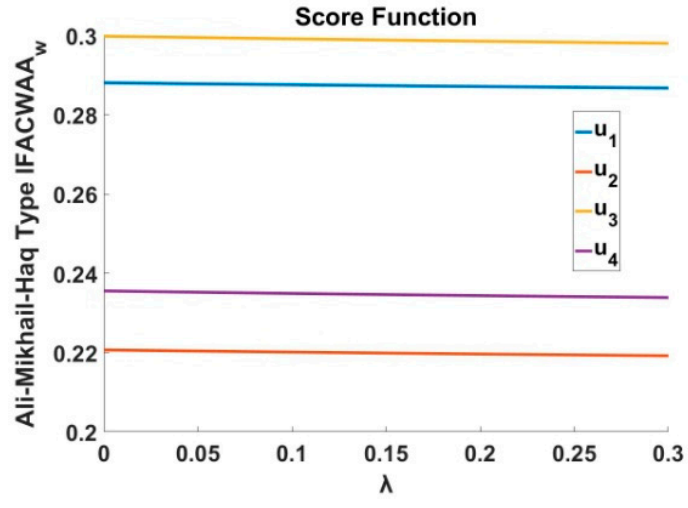

(A)

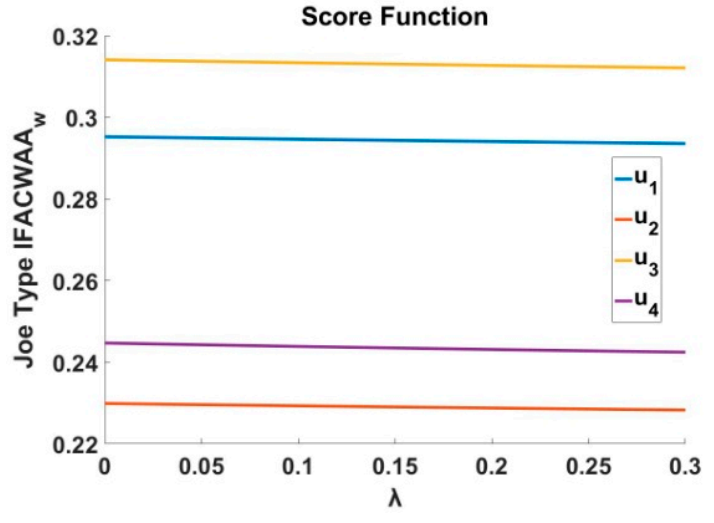

(B)

Figure 2. (A) Ali-Mikhail-Haq-type $I F A C W A A_{w}$ score result. (B) Joe-type $I F A C W A A_{w}$ score result. 


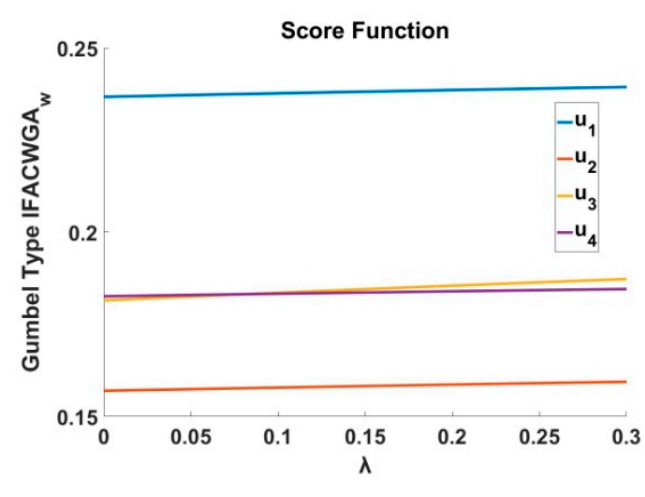

(A)

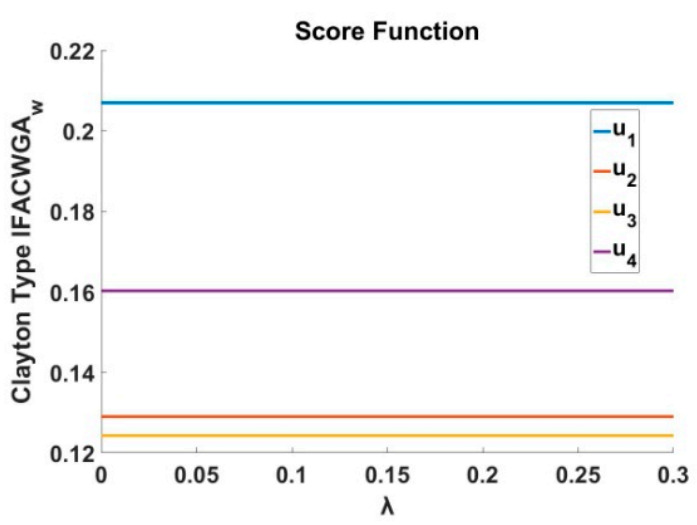

(B)

Figure 3. (A) Gumbel-type IFACWGA $A_{w}$ score result. (B) Clayton-type IFACWGA $A_{w}$ score result.

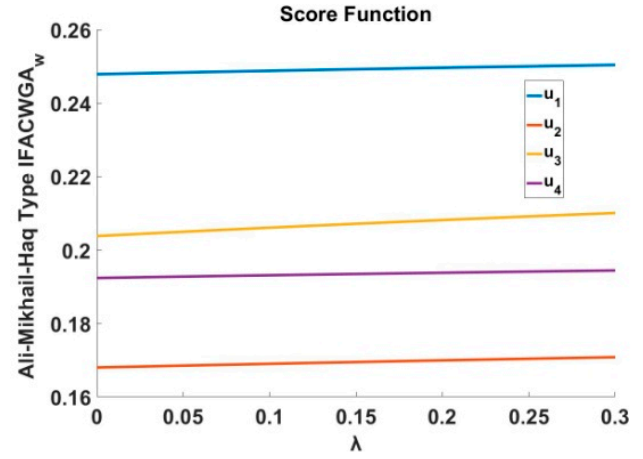

(A)

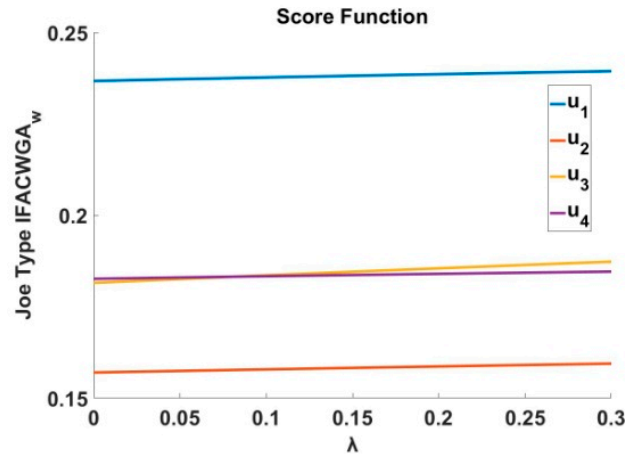

(B)

Figure 4. (A) Ali-Mikhail-Haq-type $I F A C W G A_{w}$ score result. (B) Joe-type $I F A C W G A_{w}$ score result.

\section{Discussion}

The present paper establishes specific novel operational laws of IFVs, as the generalization of the existing copula-based operational laws, with the contribution of fuzzy negations. Additionally, the paper provides the extension of the proposed operations to the arithmetic mean and to the geometric mean of IFVs.

First of all, the replacement of the $\lambda$ parameter with the numerical value zero in Sugeno class, or with the numerical value one in Yager class, in Definitions 6-8 and the corresponding tables (Tables 3-5), provides us with the operation laws and the aggregation operators of Tao et al.'s [9] work. As a result, the generalization of the existing operators by the proposed operational laws and the extension of the existing aggregation operators are verified. More specifically, the extension of the existing aggregation operators is based on the reform of the existing basic operational environment.

Regarding the provided practical example for MADM with IFVs, developed in order to point out the advantages and the flexibility of the proposed algorithm, we may call forth the following comparison with [9].

To continue with the ranking of the alternatives in [9], the usage of Gumbel-type aggregation operator suggests the third alternative $\left(u_{3}\right)$ as the most adequate one. In addition, the Clayton-, Ali-Mikhail-Haq- and Joe-type of aggregation operators provides the same alternative, respectively, through the other three alternatives are developed with a different order. On the other hand, in our approach, as Table 10 shows, there is no difference in the order of the four alternatives for each type of aggregation operator and, as a result, the order problem is distinguished.

In addition, in Table 10, the order of the alternatives is provided, suggesting the usage of IFACWGA $A_{w}$ operators for each copula type, which represents a new best alternative. Specifically, 
Gumbel-, Ali-Mikhail-Haq- and Joe-type aggregation results demonstrate that the most adequate alternative is $u_{1}$ and $u_{3}$ follows. The rest ranking remains the same with the IFACWAA $A_{w}$ operators ranking. However, in the case of Clayton-type IFACWGA $A_{w}$ ranking in respect of the three alternatives is totally different, through the first one remains $u_{1}$ demonstrating the most adequate choice. This perspective may be considered reasonable, given that the parameter $\lambda$ does not affect, neither the membership degree, nor the nonmembership degree in the aggregation process and this fact may be observed in the Clayton-type score function's plot.

Another remarkable fact about the proposed aggregation operators is that they provide two parameters affecting (in most cases) the results, suggesting more choices and flexibility for the decision makers. For the description of the affection of $\lambda$ parameter, Figures 1-4 have been utilized, as $\theta$ parameter is fixed to 1 (and -1 in the Ali-Mikhail-Haq case).

In Figure 1, apparently the Gumbel- and Clayton-type of IFACWAA $A_{w}$ operators suggest distinct score functions for each alternative, with $u_{3}$ always representing the most adequate choice and $u_{2}$ the least adequate one. The same holds for the Joe- and Ali-Mikhail-Haq-type of IFACWAA $w$ operators, as it is depicted in Figure 2. In Figure 3, the Gumbel-type of IFACWGA $A_{w}$ suggests $u_{1}$ as the most adequate choice and $u_{2}$ the least adequate one, through alternatives $u_{3}$ and $u_{4}$ that meet a crossover point. In Figure 4, we may observe that Joe-type of IFACWGA $A_{w}$ proposes the same alternatives, respectively, as the Gumbel-type approach, but also a crossover point for the alternatives $u_{3}$ and $u_{4}$.

\section{Conclusions}

The paper cited the generalization of copula-based operations of Intuitionistic Fuzzy Values (IFVs) via fuzzy negations. Additionally, novel aggregation operators were produced from the new operations of IFVs, with their properties being further investigated. As a result, an algorithm is suggested, which may be utilized in Multiple Attribute Decision Making (MADM) processes.

The main advantage of our work is that the aggregation operators of IFVs provided in the suggested algorithm are univariate parametric, therefore various intuitionistic fuzzy Archimedean copula weighted arithmetic and geometric operators could be obtained, with each one potentially being more appropriate for the decision makers. Combined with the most appropriate copula [23] for each MADM case, more specified aggregator operators would be provided to express more accurately a decision maker's attitude.

In the future our aim is to combine the proposed operations with fuzzy negations via conic sections [36], in order to produce modified families of aggregator operations of IFVs. Furthermore, we are willing to adapt the proposed operations, following the appropriate transformation, in other types of fuzzy sets, such as hesitant fuzzy sets [37], and unbalanced linguistic term sets [38] and neutrosophic sets [39], in order to construct algorithms for multiple attribute decision making and multiple attribute group decision making.

Author Contributions: Investigation, S.G. and B.P.; supervision, B.P. All authors have read and agreed to the published version of manuscript.

Funding: This research received no external funding.

Acknowledgments: The authors are very thankful to the Editor and the Referees for their corrections, valuable comments and suggestions in order to improve the quality of the present paper.

Conflicts of Interest: The authors declare no conflict of interest.

\section{References}

1. Atanassov, K.T. Intuitionistic fuzzy sets. Fuzzy Sets Syst. 1986, 20, 87-96. [CrossRef]

2. Zadeh, L.A. Fuzzy sets. Inf. Control 1965, 8, 338-353. [CrossRef]

3. Atanassov, K.T. New operations defined over the intuitionistic fuzzy sets. Fuzzy Sets Syst. 1994, 61, 137-142. [CrossRef] 
4. Beliakov, G.; Bustince, H.; Goswami, D.P.; Mukherjee, U.K.; Pal, N.R. On averaging operators for Atanassov's intuitionistic fuzzy sets. Inf. Sci. 2011, 181, 1116-1124. [CrossRef]

5. Zhao, X.; Wei, G. Some intuitionistic fuzzy Einstein hybrid aggregation operators and their application to multiple attribute decision making. Knowl. Based Syst. 2013, 37, 472-479. [CrossRef]

6. Atanassov, K.; Pasi, G.; Yager, R. Intuitionistic fuzzy interpretations of multi-criteria multi-person and multi-measurement tool decision making. Int. J. Syst. Sci. 2005, 36, 859-868. [CrossRef]

7. Wang, J.Q.; Li, J.J. Multi-criteria fuzzy decision-making method based on cross entropy and score functions. Expert Syst. Appl. 2011, 38, 1032-1038. [CrossRef]

8. Ouyang, Y.; Pedrycz, W. A new model for intuitionistic fuzzy multi-attributes decision making. Eur. J. Oper. Res. 2016, 249, 677-682. [CrossRef]

9. Tao, Z.; Han, B.; Chen, H. On intuitionistic fuzzy copula aggregation operators in multiple-attribute decision making. Cogn. Comput. 2018, 10, 610-624. [CrossRef]

10. Liu, P.; Li, H. Interval- Valued Intuitionistic Fuzzy Power Bonferroni Aggregation Operators and Their Application to Group Decission Making. Cogn. Comput. 2017, 9, 494-512. [CrossRef]

11. Seikh, M.R.; Mandal, U. Intuitionistic fuzzy Dombi aggregation operators and their application to multiple attribute decision-making. Granul. Comput. 2019, 1-16. [CrossRef]

12. Xian, S.; Guo, H.; Chai, J. Intuitionistic fuzzy linguistic induced generalized hybrid weighted averaging operator and its application to take targeted measures in poverty alleviation. Comput. Appl. Math. 2019, 38, 134. [CrossRef]

13. Shi, M.; Yang, F.; Xiao, Y. Intuitionistic fuzzy power geometric Heronian mean operators and their application to multiple attribute decision making. J. Intell. Fuzzy Syst. 2019, 37, 2651-2669. [CrossRef]

14. Zou, X.Y.; Chen, S.M.; Fan, K.Y. Multiple attribute decision making using improved intuitionistic fuzzy weighted geometric operators of intuitionistic fuzzy values. Inf. Sci. 2020, 535, 242-253. [CrossRef]

15. Xia, M.; Xu, Z.; Zhu, B. Some issues on intuitionistic fuzzy aggregation operators based on Archimedean t-conorm and t-norm. Knowl. Based Syst. 2012, 31, 78-88. [CrossRef]

16. Deschrijver, G.; Kerre, E.E. A generalization of operators on intuitionistic fuzzy sets using triangular norms and conorms. Notes Intuit. Fuzzy Sets 2002, 8, 19-27.

17. Liu, H.W.; Wang, G.J. Multi-criteria decision-making methods based on intuitionistic fuzzy sets. Eur. J. Oper. Res. 2007, 179, 220-233. [CrossRef]

18. Li, D.F. Multiattribute decision making method based on generalized OWA operators with intuitionistic fuzzy sets. Expert Syst. Appl. 2010, 37, 8673-8678. [CrossRef]

19. Yu, X.; Xu, Z. Prioritized intuitionistic fuzzy aggregation operators. Inf. Fusion 2013, 14, 108-116. [CrossRef]

20. Liu, P.; Liu, Y. An approach to multiple attribute group decision making based on intuitionistic trapezoidal fuzzy power generalized aggregation operator. Int. J. Comput. Intell. Syst. 2014, 7, 291-304. [CrossRef]

21. Ye, J. Intuitionistic fuzzy hybrid arithmetic and geometric aggregation operators for the decision-making of mechanical design schemes. Appl. Intell. 2017, 47, 73-751. [CrossRef]

22. Sirbiladze, G.; Sikharulidze, A. Extensions of probability intuitionistic fuzzy aggregation operators in fuzzy MCDM/MADM. Int. J. Inf. Technol. Decis. Mak. 2018, 17, 621-655. [CrossRef]

23. Näther, W. Copulas and t-norms: Mathematical tools for combining probabilistic information, with application to error propagation and interaction. Struct. Saf. 2010, 32, 366-371. [CrossRef]

24. Nelsen, R.B. An Introduction to Copulas, 2nd ed.; Springer Science \& Business Media: New York, NY, USA, 2006.

25. Alsina, C.; Frank, M.J.; Schweizer, B. Associative Functions: Triangular Norms and Copulas; World Scientific: Singapore, 2006.

26. Beliakov, G.; Pradera, A.; Calvo, T. Aggregation Functions: A Guide for Practitioners; Springer: Berlin/Heidelberg, Germany, 2007.

27. Massanet, S.; Pradera, A.; Ruiz-Aguilera, D.; Torrens, J. Equivalence and characterization of probabilistic and survival implications. Fuzzy Sets Syst. 2019, 359, 63-79. [CrossRef]

28. Sklar, A. Fonctions de répartition à n dimensions et leurs marges. Publ. Inst. Statist. Univ. Paris 1959, 8, $229-231$.

29. Cherubini, U.; Luciano, E.; Vecchiato, W. Copula Methods in Finance; John Wiley \& Sons, Ltd.: Chichester, UK, 2004.

30. Fréchet, M. Sur les tableaux de correlation dont les marges sont données. Comptes Rendus Hebd. Des. Seances De L Acad. Des. Sci. 1956, 242, 2426-2428.

31. Hoeffding, W. Masstabinvariante Korrelationstheorie; Schriften des Mathematischen Instituts und Instituts fur Angewandte Mathematik der Universitat Berlin: Berlin, Germany, 1940; Volume 5, pp. 181-233. 
32. Baczynski, M.; Jayaram, B. Fuzzy Implications; Springer: Berlin/Heidelberg, Germany, 2008.

33. Chen, S.M.; Tan, J.M. Handling multicriteria fuzzy decision-making problems based on vague set theory. Fuzzy Sets Syst. 1994, 67, 163-172. [CrossRef]

34. Hong, D.H.; Choi, C.H. Multicriteria fuzzy decision-making problems based on vague set theory. Fuzzy Sets Syst. 2000, 114, 103-113. [CrossRef]

35. Xu, Z.; Yager, R.R. Some geometric aggregation operators based on intuitionistic fuzzy sets. Int. J. Gen. Syst. 2006, 35, 417-433. [CrossRef]

36. Souliotis, G.; Papadopoulos, B. An algorithm for Producing Fuzzy Negations via Conical Sections. Algorithms 2019, 12, 89. [CrossRef]

37. Torra, V. Hesitant fuzzy sets. Int. J. Intell. Syst. 2010, 25, 529-539. [CrossRef]

38. Tao, Z.; Han, B.; Zhou, L.; Chen, H. The Novel Computational Model of Unbalanced Linguistic Variables Based on Archimedean Copula. Int. J. Uncertain. Fuzziness Knowl. Based Syst. 2018, 26, 601-631. [CrossRef]

39. Smarandache, F. Neutrosophic set-a generalization of the intuitionistic fuzzy set. Int. J. Pure Appl. Math. 2005, 24, 287.

(C) 2020 by the authors. Licensee MDPI, Basel, Switzerland. This article is an open access article distributed under the terms and conditions of the Creative Commons Attribution (CC BY) license (http://creativecommons.org/licenses/by/4.0/). 